J. DIFFERENTIAL GEOMETRY

84 (2010) 127-161

\title{
SELF-SIMILAR SOLUTIONS AND TRANSLATING SOLITONS FOR LAGRANGIAN MEAN CURVATURE FLOW
}

\author{
Dominic Joyce, Yng-Ing Lee \& MaO-Pei Tsui
}

\begin{abstract}
We construct many self-similar and translating solitons for Lagrangian mean curvature flow, including self-expanders and translating solitons with arbitrarily small oscillation on the Lagrangian angle. Our translating solitons play the same role as cigar solitons in Ricci flow, and are important in studying the regularity of Lagrangian mean curvature flow.

Given two transverse Lagrangian planes $\mathbb{R}^{n}$ in $\mathbb{C}^{n}$ with sum of characteristic angles less than $\pi$, we show there exists a Lagrangian self-expander asymptotic to this pair of planes. The Maslov class of these self-expanders is zero. Thus they can serve as local models for surgeries on Lagrangian mean curvature flow. Families of self-shrinkers and self-expanders with different topologies are also constructed. This paper generalizes the work of Anciaux [1], Joyce [12], Lawlor [15], and Lee and Wang [18, 19].
\end{abstract}

\section{Introduction}

Special Lagrangian submanifolds in Calabi-Yau $n$-folds have received much attention in recent years, as they are key ingredients in the Strominger-Yau-Zaslow Conjecture [25], which explains Mirror Symmetry of Calabi-Yau 3-folds. Thomas and Yau [26] defined a notion of stability for graded Lagrangians $L$ in a Calabi-Yau $n$-fold $M$, and conjectured that if $L$ is stable then the Lagrangian mean curvature flow of $L$ exists for all time and converges to a special Lagrangian submanifold $L_{\infty}$ in $M$, which should be the unique special Lagrangian in the Hamiltonian equivalence class of $L$.

Rewriting this in terms of the derived Fukaya category $D^{b} \operatorname{Fuk}(M)$ of $M$, as in Kontsevich's Homological Mirror Symmetry program [14], and using Bridgeland's notion of stability condition on triangulated categories [4], one can state an improved (but still over-simplified) version of the Thomas-Yau conjecture as follows: for any Calabi-Yau $n$-fold $M$, there should exist a Bridgeland stability condition $(Z, \mathcal{P})$ on $D^{b} \mathrm{Fuk}(M)$ depending on the holomorphic $(n, 0)$-form $\Omega$ on $M$, such that a graded Lagrangian $L$ in $M$ is $(Z, \mathcal{P})$-stable, regarded as an object in $D^{b} \operatorname{Fuk}(M)$, 
if and only if the Lagrangian mean curvature flow of $L$ exists for all time and converges to a special Lagrangian submanifold $L_{\infty}$ in $M$, which should be unique in the isomorphism class of $L$ in $D^{b} \operatorname{Fuk}(M)$. A related method for constructing special Lagrangians by minimizing volume amongst Lagrangians using Geometric Measure Theory was proposed by Schoen and Wolfson [23].

To carry these programs through to their conclusion will require a deep understanding of Lagrangian mean curvature flow, and of the possible singularities that can occur during it in finite time. Singularities in Lagrangian mean curvature flow are generally locally modelled on soliton solutions, such as Lagrangians in $\mathbb{C}^{n}$ which are moved by rescaling or translation by mean curvature flow. There are two important results in this area. The first one is due to Wang [27], who observed that mean curvature flow for almost calibrated Lagrangians in Calabi-Yau $n$-folds cannot develop type I singularities. And the second one is due to Neves [20], who (loosely) proved that singularities of such flows are modelled to leading order on special Lagrangian cones when applying central blow up near the singularities.

In this paper, we construct many examples of self-similar solutions and translating solitons for Lagrangian mean curvature flow. Our Lagrangians $L$ in $\mathbb{C}^{n}$ are the total space of a 1-parameter family $Q_{s}, s \in I$, where $I$ is an open interval in $\mathbb{R}$, and each $Q_{s}$ is a quadric in a Lagrangian plane $\mathbb{R}^{n}$ in $\mathbb{C}^{n}$, which evolve according to an o.d.e. in $s$. The construction includes and generalizes examples of Lagrangian solitons or special Lagrangians due to Anciaux [1], Joyce [12], Lawlor [15], and Lee and Wang $[\mathbf{1 8}, \mathbf{1 9}]$.

The authors believe that two of our families of examples may have particular significance for future work on Lagrangian mean curvature flow. Firstly, in Theorems $\mathrm{C}$ and $\mathrm{D}$ of $\S 3.2$, we show that if $L_{1}, L_{2}$ are transverse Lagrangian planes in $\mathbb{C}^{n}$ and the sum of characteristic angles of $L_{1}, L_{2}$ is less than $\pi$, and $\alpha>0$, then we can construct a unique closed, embedded Lagrangian self-expander $L$ with rate $\alpha$ diffeomorphic to $\mathcal{S}^{n-1} \times \mathbb{R}$ and asymptotic to $L_{1} \cup L_{2}$. These examples could be used as local models for surgeries during Lagrangian mean curvature flow.

As in the Ricci flow proof of the Poincaré conjecture [22], it seems likely that to get long-time existence for Lagrangian mean curvature flow, it will be necessary to allow the flow to develop singularities, and continue the flow after a surgery which changes the topology of the Lagrangian. Research by the first author (unpublished) indicates that an important condition in the improved Thomas-Yau conjecture described above is that the Lagrangians should have unobstructed Lagrangian Floer homology, in the sense of Fukaya, Oh, Ohta and Ono $[6,7]$. But mean curvature flow amongst nonsingular, immersed Lagrangians can cross 'walls', on the other side of which Lagrangian Floer 
homology is obstructed. When this happens, the correct thing to do is to do a surgery, and glue in a Lagrangian self-expander from Theorems $\mathrm{C}$ and D.

Secondly, in Corollary I of $\S 3.4$ we give an explicit family of closed, embedded Lagrangian translating solitons $L$ in $\mathbb{C}^{n}$ for $n \geqslant 2$, which are diffeomorphic to $\mathbb{R}^{n}$, and asymptotic in a weak sense to a union $L_{1} \cup L_{2}$ of Lagrangian planes $L_{1}, L_{2} \cong \mathbb{R}^{n}$ in $\mathbb{C}^{n}$, with $L_{1} \cap L_{2} \cong \mathbb{R}$. The oscillation of the Lagrangian angle of $L$ can be chosen arbitrarily small. If these examples can arise as local models for finite time singularities for Lagrangian mean curvature flow, they may represent a kind of bad behavior, which could cause difficulties with the Thomas-Yau program even in dimension 2.

As well as these two families, we construct new examples of compact, immersed Lagrangian self-shrinkers in $\mathbb{C}^{n}$ diffeomorphic to $\mathcal{S}^{1} \times \mathcal{S}^{n-1}$, of closed, immersed Lagrangian self-expanders and self-shrinkers diffeomorphic to $\mathcal{S}^{1} \times \mathcal{S}^{m-1} \times \mathbb{R}^{n-m}$ for $0<m<n$, of non-closed, immersed Lagrangian self-expanders diffeomorphic to $\mathcal{S}^{m} \times \mathbb{R}^{n-m}$ for $0<m<n-1$, of non-closed, immersed Lagrangian self-shrinkers diffeomorphic to $\mathcal{S}^{m} \times \mathbb{R}^{n-m}$ for $0<m<n$, and of closed, embedded Lagrangian translating solitons diffeomorphic to $\mathbb{R}^{n}$ with infinite oscillation of the Lagrangian angle. These examples include those of Anciaux $[\mathbf{1}]$ and Lee and Wang $[\mathbf{1 8}, \mathbf{1 9}]$, and approach the special Lagrangians of Joyce $[\mathbf{1 2}]$ and Lawlor $[\mathbf{1 5}]$ in a limit.

We begin in $\S 2$ with some background material. Our main results are stated and discussed in $\S 3$, which is the part of the paper we intend most people to actually read. The proofs are given in $\S 4-\S 6$.

Acknowledgements. Part of this paper was completed while the authors were visiting Taida Institute of Mathematical Sciences (TIMS) in National Taiwan University, Taipei, Taiwan. The authors wish to express their gratitude for the excellent support provided by the center during their stays. The first author would like to thank Richard Thomas and Tom Ilmanen for useful conversations. The second author would like to express her special gratitude to $\mathrm{Mu}$-Tao Wang for many enlightening discussions and collaborations on the subject over the years, which benefitted her a lot. The calculation techniques used in the proof of Theorem A were first observed in $[\mathbf{1 8}, \mathbf{1 9}]$. She would also like to thank André Neves for his interests in this work and enlightening discussions. The third author would like to thank Duong Hong Phong, Shing-Tung Yau and Mu-Tao Wang for their constant advice, encouragement, and support.

\section{Background material}

Our ambient space is always the complex Euclidean space $\mathbb{C}^{n}$ with coordinates $z_{j}=x_{j}+i y_{j}$, the standard symplectic form $\omega=\sum_{j=1}^{n} \mathrm{~d} x_{j} \wedge$ 
$\mathrm{d} y_{j}$, and the standard almost complex structure $J$ with $J\left(\frac{\partial}{\partial x_{j}}\right)=\frac{\partial}{\partial y_{j}}$. A Lagrangian submanifold is an $n$-dimensional submanifold in $\mathbb{C}^{n}$ on which the symplectic form $\omega$ vanishes. On a Lagrangian submanifold $L$, the mean curvature vector $H$ is given by

$$
H=J \nabla \theta,
$$

where $\theta$ is the Lagrangian angle and $\nabla$ is the gradient on $L$. The angle function $\theta: L \rightarrow \mathbb{R}$ or $\theta: L \rightarrow \mathbb{R} / 2 \pi \mathbb{Z}$ can be defined by the relation that $\left.\mathrm{d} z_{1} \wedge \cdots \wedge \mathrm{d} z_{n}\right|_{L} \equiv e^{i \theta} \operatorname{vol}_{L}$. When $\cos \theta \geq \epsilon$ on $L$ for some positive $\epsilon>0, L$ is called almost calibrated. The Maslov class on $L$ is defined by the cohomology class of $\mathrm{d} \theta$. Hence $L$ is Maslov zero when $\theta$ is a globally defined function from $L$ to $\mathbb{R}$.

By the first variation formula, the mean curvature vector points in the direction in which the volume decreases most rapidly. Mean curvature flow deforms the submanifold in the direction of the mean curvature vector. As special Lagrangians are volume minimizing, it is natural to use mean curvature flow to construct special Lagrangians. Equation (1) implies that mean curvature flow is a Lagrangian deformation, that is, a Lagrangian submanifold remains Lagrangian under mean curvature flow, as in Smoczyk [24].

A Lagrangian submanifold $L$ in $\mathbb{C}^{n}$ is fixed by mean curvature flow if and only if the Lagrangian angle $\theta$ on $L$ is constant, that is, if and only if $L$ is special Lagrangian with phase $e^{i \theta}$, as in Harvey and Lawson $[\mathbf{9}, \S \mathrm{III}]$. A Lagrangian $L$ in $\mathbb{C}^{n}$ is called Hamiltonian stationary if the Lagrangian angle $\theta$ on $L$ is harmonic, that is, if $\Delta \theta=0$ on $L$. This implies that the volume of $L$ is stationary under Hamiltonian deformations.

In geometric flows such as Ricci flow or mean curvature flow, singularities are often locally modelled on soliton solutions. In the case of mean curvature flows, two types of soliton solutions of particular interest are those moved by scaling or translation in Euclidean space. We recall that solitons moved by scaling must be of the form:

Definition 2.1. A submanifold $L$ in Euclidean space $\mathbb{R}^{n}$ is called a self-similar solution if $H \equiv \alpha F^{\perp}$ on $L$ for some constant $\alpha$ in $\mathbb{R}$, where $F^{\perp}$ is the projection of the position vector $F$ in $\mathbb{R}^{n}$ to the normal bundle of $L$, and $H$ is the mean curvature vector of $L$ in $\mathbb{R}^{n}$. It is called a self-shrinker if $\alpha<0$ and self-expander if $\alpha>0$.

It is not hard to see that if $F$ is a self-similar solution, then $F_{t}$ defined by $F_{t}=\sqrt{2 \alpha t} F$ is moved by the mean curvature flow. By Huisken's monotonicity formula [10], any central blow up of a finite-time singularity of the mean curvature flow is a self-similar solution. When $\alpha=0$, the submanifold is minimal. The submanifolds which are moved by translation along mean curvature flow must be of the form: 
Definition 2.2. A submanifold $L$ in Euclidean space $\mathbb{R}^{n}$ is called a translating soliton if there exists a constant vector $T$ in $\mathbb{R}^{n}$ such that $H+V \equiv T$ on $L$, where $V$ is the component of $T$ tangent to $L$, and $H$ is the mean curvature vector of $L$ in $\mathbb{R}^{n}$. An equivalent equation is $H \equiv T^{\perp}$. The 1-parameter family of submanifolds $L_{t}$ defined by $L_{t}=L+t T$ for $t \in \mathbb{R}$ is then a solution to mean curvature flow, and we call $T$ a translating vector.

Definition 2.3. A translating soliton is called a gradient translating soliton if $V=\nabla f$ for some smooth function $f: L \rightarrow \mathbb{R}$.

Any translating soliton for mean curvature flow in $\mathbb{R}^{n}$ must be a gradient translating soliton. Since this simple fact does not appear in the literature, we include a proof here for completeness.

Proposition 2.4. A translating soliton in $\mathbb{R}^{n}$ that satisfies $H+V=$ $T$ where $T$ is a constant vector must be a gradient translating soliton. In fact, $H+\nabla\langle T, F\rangle=T$, where $F$ is the position vector.

Proof. Let $F=\left(F_{1}, \ldots, F_{n}\right)$ be the position function and $\bar{\nabla}$ be the standard connection in $\mathbb{R}^{n}$. Then $\bar{\nabla} F_{i}=e_{i}$. We may write $T=$ $\sum_{i=1}^{n} T^{i} e_{i}=\bar{\nabla}\langle T, F\rangle$. Then $(\bar{\nabla}\langle T, F\rangle)^{\top}=V$ and $\left.\nabla\langle T, F\rangle\right|_{L}=V$, where $(\bar{\nabla}\langle T, F\rangle)^{\top}$ is the orthogonal projection of $\bar{\nabla}\langle T, F\rangle$ to $T L$. This shows $L$ is a gradient translating soliton.

q.e.d.

Here is a counterpart of this result for Lagrangian translating solitons.

Proposition 2.5. A connected Lagrangian $L$ in $\mathbb{C}^{n}$ is a translating soliton with translating vector $T$ if and only if $\theta \equiv-\left.\langle J T, F\rangle\right|_{L}+c$ for some $c \in \mathbb{R}$, where $F$ is the position vector. Thus a Lagrangian translating soliton is Maslov zero.

Proof. Suppose $L$ is a translating soliton with translating vector $T$. We have $J \nabla \theta \equiv H \equiv T^{\perp}$ as sections of the normal bundle of $L$ in $\mathbb{C}^{n}$. Applying $-J$ gives $\nabla \theta \equiv-J\left(T^{\perp}\right)=-(J T)^{\top}$, as sections of the tangent bundle of $L$. We then have $\nabla \theta=-\nabla\langle J T, F\rangle$ as in the proof of Proposition 2.4. Because $L$ is connected, it follows that $\theta \equiv-\left.\langle J T, F\rangle\right|_{L}+c$ for some $c \in \mathbb{R}$. Hence $\theta$ can be lifted from $\mathbb{R} / 2 \pi \mathbb{Z}$ to $\mathbb{R}$, and $L$ is Maslov zero. Conversely, suppose $\theta \equiv-\left.\langle J T, F\rangle\right|_{L}+c$. Then $\nabla \theta=-\nabla\langle J T, F\rangle=-(\bar{\nabla}\langle J T, F\rangle)^{\top}=-(J T)^{\top}$. It follows that $H=J \nabla \theta=T^{\perp}$, and $L$ is a translating soliton with vector $T$. $\quad$ q.e.d.

\section{Statements of main results}

We now state and briefly discuss the main results of this paper. The results of $\S 3.1, \S 3.2$, and $\S 3.3$ will be proved in sections 4,5 , and 6 , respectively. The proofs of results in $\S 3.4$ are brief, and are included there. 
3.1. An ansatz for self-similar Lagrangians. The following ansatz describes the class of $n$-submanifolds of $\mathbb{C}^{n}$ amongst which we will seek examples self-similar solutions for Lagrangian mean curvature flow.

Ansatz 3.1. Fix $n \geqslant 1$. Consider $n$-submanifolds $L$ in $\mathbb{C}^{n}$ of the form

$$
L=\left\{\left(x_{1} w_{1}(s), \ldots, x_{n} w_{n}(s)\right): s \in I, x_{j} \in \mathbb{R}, \sum_{j=1}^{n} \lambda_{j} x_{j}^{2}=C\right\},
$$

where $\lambda_{1}, \ldots, \lambda_{n}, C \in \mathbb{R} \backslash\{0\}$ are nonzero constants, $I$ is an open interval in $\mathbb{R}$, and $w_{1}, \ldots, w_{n}: I \rightarrow \mathbb{C} \backslash\{0\}$ are smooth. We want $L$ to satisfy:

(i) $L$ is Lagrangian;

(ii) the Lagrangian angle $\theta: L \rightarrow \mathbb{R}$ or $\theta: L \rightarrow \mathbb{R} / 2 \pi \mathbb{Z}$ of $L$ is a function only of $s$, not of $x_{1}, \ldots, x_{n}$; and

(iii) $L$ is a self-similar solution under mean curvature flow in $\mathbb{C}^{n}$, that is, $H \equiv \alpha F^{\perp}$ on $L$ as in Definition 2.1.

The motivation for this ansatz is that it includes, and generalizes, several families of examples in the literature. For special Lagrangian submanifolds, with $\theta \equiv 0$ in (ii) and $\alpha=0$ in (iii), the ansatz includes the examples of Lawlor [15] with $\lambda_{1}=\cdots=\lambda_{n}=C=1$, and Joyce [12, $\S 5-\S 6]$. For more general Lagrangian self-similar solutions, it includes the examples of Abresch and Langer [2] when $n=1$, the examples of Anciaux [1], which have $\lambda_{1}=\cdots=\lambda_{n}=C=1$ and are symmetric under the action of $\mathrm{SO}(n)$ on $\mathbb{C}^{n}$, and the examples of Lee and Wang $[\mathbf{1 8}, \S 6],[\mathbf{1 9}]$, which have $w_{j}(s) \equiv e^{i \lambda_{j} s}$. M.-T. Wang and the second author also tried to study an ansatz of a similar form to (2) before.

It is a long but straightforward calculation to find the conditions on $\lambda_{1}, \ldots, \lambda_{n}, C, w_{1}, \ldots, w_{n}$ for $L$ in (2) to be Lagrangian, to compute its Lagrangian angle $\theta$ and mean curvature $H$, and to work out whether $L$ is a self-similar solution to Lagrangian mean curvature flow. In this way we prove the following theorem.

Theorem A. Let $\lambda_{1}, \ldots, \lambda_{n}, C \in \mathbb{R} \backslash\{0\}$ and $\alpha \in \mathbb{R}$ be constants, $I$ be an open interval in $\mathbb{R}$, and $\theta: I \rightarrow \mathbb{R}$ or $\theta: I \rightarrow \mathbb{R} / 2 \pi \mathbb{Z}$ and $w_{1}, \ldots, w_{n}: I \rightarrow \mathbb{C} \backslash\{0\}$ be smooth functions. Suppose that

$$
\begin{aligned}
\frac{\mathrm{d} w_{j}}{\mathrm{~d} s} & =\lambda_{j} e^{i \theta(s)} \overline{w_{1} \cdots w_{j-1} w_{j+1} \cdots w_{n}}, \quad j=1, \ldots, n, \\
\frac{\mathrm{d} \theta}{\mathrm{d} s} & =\alpha \operatorname{Im}\left(e^{-i \theta(s)} w_{1} \cdots w_{n}\right),
\end{aligned}
$$

hold in I. Then the submanifold $L$ in $\mathbb{C}^{n}$ given by

$$
L=\left\{\left(x_{1} w_{1}(s), \ldots, x_{n} w_{n}(s)\right): s \in I, x_{j} \in \mathbb{R}, \sum_{j=1}^{n} \lambda_{j} x_{j}^{2}=C\right\},
$$

is Lagrangian, with Lagrangian angle $\theta(s)$ at $\left(x_{1} w_{1}(s), \ldots, x_{n} w_{n}(s)\right)$, and its position vector $F$ and mean curvature vector $H$ satisfy $\alpha F^{\perp}=$ $C H$. That is, $L$ is a self-expander when $\alpha / C>0$ and a self-shrinker when $\alpha / C<0$. When $\alpha=0$ the Lagrangian angle $\theta$ is constant, so 
that $L$ is special Lagrangian, with $H=0$. In this case the construction reduces to that of Joyce $[\mathbf{1 2}, \S 5]$.

We can simplify the equations (3), generalizing [12, §5.2].

Theorem B. In the situation of Theorem $A$, let $w_{1}, \ldots, w_{n}, \theta$ satisfy (3). Write $w_{j} \equiv r_{j} e^{i \phi_{j}}$ and $\phi=\sum_{j=1}^{n} \phi_{j}$, for functions $r_{j}: I \rightarrow(0, \infty)$ and $\phi_{1}, \ldots, \phi_{n}, \phi: I \rightarrow \mathbb{R}$ or $\mathbb{R} / 2 \pi \mathbb{Z}$. Fix $s_{0} \in I$. Define $u: I \rightarrow \mathbb{R}$ by

$$
u(s)=2 \int_{s_{0}}^{s} r_{1}(t) \cdots r_{n}(t) \cos (\phi(t)-\theta(t)) \mathrm{d} t .
$$

Then $r_{j}^{2}(s) \equiv \alpha_{j}+\lambda_{j} u(s)$ for $j=1, \ldots, n$ and $s \in I$, where $\alpha_{j}=r_{j}^{2}\left(s_{0}\right)$. Define a degree $n$ polynomial $Q(u)$ by $Q(u)=\prod_{j=1}^{n}\left(\alpha_{j}+\lambda_{j} u\right)$. Then the system of equations (3) can be rewritten as

$$
\left\{\begin{aligned}
\frac{\mathrm{d} u}{\mathrm{~d} s} & =2 Q(u)^{1 / 2} \cos (\phi-\theta), \\
\frac{\mathrm{d} \phi_{j}}{\mathrm{~d} s} & =-\frac{\lambda_{j} Q(u)^{1 / 2} \sin (\phi-\theta)}{\alpha_{j}+\lambda_{j} u}, \quad j=1, \ldots, n, \\
\frac{\mathrm{d} \phi}{\mathrm{d} s} & =-Q(u)^{1 / 2}(\ln Q(u))^{\prime} \sin (\phi-\theta), \\
\frac{\mathrm{d} \theta}{\mathrm{d} s} & =\alpha Q(u)^{1 / 2} \sin (\phi-\theta) .
\end{aligned}\right.
$$

The Lagrangian self-similar solution $L$ in Theorem A may be rewritten

$$
\begin{array}{r}
L=\left\{\left(x_{1} \sqrt{\alpha_{1}+\lambda_{1} u(s)} e^{i \phi_{1}(s)}, \ldots, x_{n} \sqrt{\alpha_{n}+\lambda_{n} u(s)} e^{i \phi_{n}(s)}\right):\right. \\
\left.x_{1}, \ldots, x_{n} \in \mathbb{R}, s \in I, \sum_{j=1}^{n} \lambda_{j} x_{j}^{2}=C\right\} .
\end{array}
$$

Moreover, for some $A \in \mathbb{R}$ the equations (5) have the first integral

$$
Q(u)^{1 / 2} e^{\alpha u / 2} \sin (\phi-\theta) \equiv A .
$$

Remark 3.2. There is a lot of freedom to rescale the constants in Theorems A and B without changing the Lagrangian $L$. In particular: (a) Set

$$
\begin{gathered}
\tilde{\lambda}_{j}=C \lambda_{j} /\left|C \lambda_{j}\right|, \quad \tilde{C}=1, \quad \tilde{\alpha}=\alpha / C, \quad \tilde{I}=C|C|^{-n / 2} \prod_{j=1}^{n}\left|\lambda_{j}\right|^{1 / 2} \cdot I, \\
\tilde{s}=C|C|^{-n / 2} \prod_{j=1}^{n}\left|\lambda_{j}\right|^{1 / 2} s, \tilde{\theta}=\theta, \quad \tilde{w}_{j}=|C|^{1 / 2}\left|\lambda_{j}\right|^{-1 / 2} w_{j}, \\
\tilde{x}_{j}=|C|^{-1 / 2}\left|\lambda_{j}\right|^{1 / 2} x_{j}, \quad \tilde{r}_{j}=|C|^{1 / 2}\left|\lambda_{j}\right|^{-1 / 2} r_{j}, \quad \tilde{\phi}_{j}=\phi_{j}, \quad \tilde{\phi}=\phi, \\
\tilde{u}=C u, \quad \tilde{\alpha}_{j}=|C|\left|\lambda_{j}\right|^{-1} \alpha_{j}, \quad \tilde{A}=|C|^{n / 2} \prod_{j=1}^{n}\left|\lambda_{j}\right|^{-1 / 2} A,
\end{gathered}
$$

where we regard $\tilde{w}_{j}, \tilde{\theta}, \tilde{r}_{j}, \tilde{\phi}_{j}, \tilde{\phi}, \tilde{u}$ as functions of $\tilde{s}$ rather than $s$, so that $\tilde{w}_{j}(\tilde{s})=|C|^{1 / 2}\left|\lambda_{j}\right|^{-1 / 2} w_{j}\left(C^{-1}|C|^{n / 2} \prod_{j=1}^{n}\left|\lambda_{j}\right|^{-1 / 2} \tilde{s}\right)$, for instance. Then these $\tilde{\lambda}_{1}, \ldots, \tilde{A}$ satisfy Theorems $\mathrm{A}$ and $\mathrm{B}$, with the same Lagrangian $L$. Thus without loss of generality we can suppose $\lambda_{j}= \pm 1$ for all $j$ and $C=1$. 
(b) Translations of $I$ in $\mathbb{R}$, so that $I \mapsto I+c, s \mapsto s+c$ also do not change $L$. Thus we can fix $0 \in I$ and $s_{0}=0$ in Theorem B.

(c) Changing $s \mapsto-s$ and $\theta \mapsto \theta+\pi$ gives a solution with the same $L$, but the opposite orientation.

(d) Changing $w_{1} \mapsto-w_{1}, s \mapsto-s, \phi_{1} \mapsto \phi_{1}+\pi, \phi \mapsto \phi+\pi$, and $A \mapsto-A$ gives a solution with the same $L$. When $A=0$, as $Q(u)=r_{1} \cdots r_{n}>0$ equation (7) gives $\sin (\phi-\theta) \equiv 0$, so (5) implies that $\phi_{j}, \phi, \theta$ are constant. Thus $L$ is an open subset of the special Lagrangian $n$-plane

$$
\left\{\left(e^{i \phi_{1}} x_{1}, \ldots, e^{i \phi_{n}} x_{n}\right): x_{1}, \ldots, x_{n} \in \mathbb{R}\right\}
$$

in $\mathbb{C}^{n}$. As we are not interested in this case, we will either take $A>0$ or $A<0$. For the case of explicit Lagrangian self-expanders in $\S 5$, we choose $A<0$ to make the solutions have a similar expression to Lawlor's examples, while we take $A>0$ in $\S 6$.

(e) If $t>0$, changing $\alpha \mapsto t^{-2} \alpha, I \mapsto t^{n-2} I, s \mapsto t^{n-2} s, w_{j} \mapsto t w_{j}$, $r_{j} \mapsto t r_{j}, u \mapsto t^{2} u, \alpha_{j} \mapsto t^{2} \alpha_{j}, A \mapsto t^{n} A$ gives another solution for the Lagrangian $t L$ rather than $L$. Thus, by allowing rescalings $L \mapsto t L$, we can also set $\alpha$ to $1,-1$, or 0 . But we shall retain the parameter $\alpha$, since taking limits $\alpha \rightarrow 0$ shows how our Lagrangian self-expanders or self-shrinkers are related to special Lagrangian examples.

3.2. A class of explicit Lagrangian self-expanders. As in Remark 3.2 , in Theorems $\mathrm{A}$ and $\mathrm{B}$ we may without loss of generality suppose that $\lambda_{j}= \pm 1, C=1$ and $A<0$. We now consider the case in which $\lambda_{1}=\cdots=\lambda_{n}=C=1, \alpha \geqslant 0$ and $A<0$. Then the Lagrangians $L$ we get are embedded and diffeomorphic to $\mathcal{S}^{n-1} \times \mathbb{R}$. When $\alpha=0$ they are the special Lagrangian 'Lawlor necks' found by Lawlor [15] and studied by Harvey [8, p. 139-143], and Theorem C below generalizes Harvey's treatment. For $\alpha>0$ they are Lagrangian self-expanders. When $\alpha>0$, $a_{1}=\cdots=a_{n}$ and $\psi_{1}=\cdots=\psi_{n}$ in Theorem $\mathrm{C}$, the self-expander $L$ is invariant under $\mathrm{SO}(n)$, and is one of the examples found by Anciaux [1].

Theorem C. In Theorems $A$ and B, suppose that $\lambda_{1}=\cdots=\lambda_{n}=$ $C=1, \alpha \geqslant 0$ and $A<0$. Then any solution of (3), or equivalently of (5), on an interval $I$ in $\mathbb{R}$ can be extended to a unique largest open interval $I_{\max }$ in $\mathbb{R}$. Take $I=I_{\max }$. Then by changing variables from $s$ in $I_{\max }$ to $y=y(s)$ in $\mathbb{R}$, we may rewrite the Lagrangian self-expander $L$ of (4) and (6) explicitly as follows. Conversely, every $L$ of the following form comes from Theorems $A$ and $B$ with $\lambda_{1}=\cdots=\lambda_{n}=C=1, \alpha \geqslant 0$ and $A<0$.

For given constants $\alpha \geqslant 0, a_{1}, \ldots, a_{n}>0$ and $\psi_{1}, \ldots, \psi_{n} \in \mathbb{R}$, define $w_{j}(y)=e^{i \phi_{j}(y)} r_{j}(y)$ for $j=1, \ldots, n$ and $y \in \mathbb{R}$ by

$$
r_{j}(y)=\sqrt{\frac{1}{a_{j}}+y^{2}} \quad \text { and } \quad \phi_{j}(y)=\psi_{j}+\int_{0}^{y} \frac{\mathrm{d} t}{\left(\frac{1}{a_{j}}+t^{2}\right) \sqrt{P(t)}},
$$


where $P(t)=\frac{1}{t^{2}}\left(\prod_{k=1}^{n}\left(1+a_{k} t^{2}\right) e^{\alpha t^{2}}-1\right)$. Then

$$
L=\left\{\left(x_{1} w_{1}(y), \ldots, x_{n} w_{n}(y)\right): x_{1}, \ldots, x_{n} \in \mathbb{R}, \sum_{j=1}^{n} x_{j}^{2}=1\right\}
$$

is a closed, embedded Lagrangian diffeomorphic to $\mathcal{S}^{n-1} \times \mathbb{R}$ and satisfying $\alpha F^{\perp}=H$. If $\alpha>0$ it is a self-expander, and if $\alpha=0$ it is one of Lawlor's examples of special Lagrangian submanifolds [15]. It has Lagrangian angle

$$
\theta(y)=\sum_{j=1}^{n} \phi_{j}(y)+\arg \left(y+i P(y)^{-1 / 2}\right) .
$$

We can describe the asymptotic behavior of these Lagrangians:

Theorem D. In the situation of Theorem $C$, there exist $\bar{\phi}_{1}, \ldots, \bar{\phi}_{n} \in$ $\left(0, \frac{\pi}{2}\right]$ with $\bar{\phi}_{j}=\int_{0}^{\infty} \frac{\mathrm{d} t}{\left(\frac{1}{a_{j}}+t^{2}\right) \sqrt{P(t)}}$ for $j=1, \ldots, n$, such that the Lagrangian $L$ is asymptotic at infinity to the union of Lagrangian planes $L_{1} \cup L_{2}$, where

$$
\begin{aligned}
& L_{1}=\left\{\left(e^{i\left(\psi_{1}+\bar{\phi}_{1}\right)} t_{1}, \ldots, e^{i\left(\psi_{n}+\bar{\phi}_{n}\right)} t_{n}\right): t_{1}, \ldots, t_{n} \in \mathbb{R}\right\}, \\
& L_{2}=\left\{\left(e^{i\left(\psi_{1}-\bar{\phi}_{1}\right)} t_{1}, \ldots, e^{i\left(\psi_{n}-\bar{\phi}_{n}\right)} t_{n}\right): t_{1}, \ldots, t_{n} \in \mathbb{R}\right\} .
\end{aligned}
$$

We have $0<\bar{\phi}_{1}+\cdots+\bar{\phi}_{n}<\frac{\pi}{2}$ if $\alpha>0$, and $\bar{\phi}_{1}+\cdots+\bar{\phi}_{n}=\frac{\pi}{2}$ if $\alpha=0$.

Fix $\alpha>0$. Then $\Phi^{n}:\left(a_{1}, \ldots, a_{n}\right) \mapsto\left(\bar{\phi}_{1}, \ldots, \bar{\phi}_{n}\right)$ gives a diffeomorphism

$$
\Phi^{n}:(0, \infty)^{n} \longrightarrow\left\{\left(\bar{\phi}_{1}, \ldots, \bar{\phi}_{n}\right) \in\left(0, \frac{\pi}{2}\right)^{n}: 0<\bar{\phi}_{1}+\cdots+\bar{\phi}_{n}<\frac{\pi}{2}\right\} .
$$

That is, for all $\alpha>0$ and $L_{1}, L_{2}$ satisfying $0<\bar{\phi}_{1}+\cdots+\bar{\phi}_{n}<\frac{\pi}{2}$ as above, Theorem $C$ gives a unique Lagrangian expander $L$ asymptotic to $L_{1} \cup L_{2}$.

When $\alpha=0$, it is studied by Lawlor in [15]. The map $\Phi^{n}:\left(a_{1}, \ldots, a_{n}\right)$ $\mapsto\left(\bar{\phi}_{1}, \ldots, \bar{\phi}_{n}\right)$ gives a surjection

$$
\Phi^{n}:(0, \infty)^{n} \longrightarrow\left\{\left(\bar{\phi}_{1}, \ldots, \bar{\phi}_{n}\right) \in\left(0, \frac{\pi}{2}\right]^{n}: \bar{\phi}_{1}+\cdots+\bar{\phi}_{n}=\frac{\pi}{2}\right\},
$$

such that $\left(a_{1}, \ldots, a_{n}\right)$ and $\left(a_{1}^{\prime}, \ldots, a_{n}^{\prime}\right)$ have the same image $\left(\bar{\phi}_{1}, \ldots, \bar{\phi}_{n}\right)$ if and only if $a_{j}^{\prime}=t a_{j}$ for some $t>0$ and all $j=1, \ldots, n$, and the corresponding special Lagrangians $L, L^{\prime}$ satisfy $L^{\prime}=t^{-1 / 2} L$.

By applying an element of $\mathrm{U}(n)$, Theorem $\mathrm{D}$ also shows that we can construct a unique Lagrangian self-expander with constant $\alpha$ asymptotic to any pair of Lagrangian planes in $\mathbb{C}^{n}$ which intersect transversely at the origin and have sum of characteristic angles less than $\pi$. As the union of a pair of planes is volume minimizing if and only if the sum of characteristic angles is greater or equal to $\pi[\mathbf{1 5}]$, our result is sharp.

The Lagrangian self-expanders in Theorems $\mathrm{C}$ and D have arbitrarily small oscillation of the Lagrangian angle. That is, if $\sum_{j=1}^{n} \bar{\phi}_{j}=\frac{\pi}{2}-\epsilon$ in Theorem D, then (9) implies that $L$ in (8) has Lagrangian angle varying in $\left(\sum_{j=1}^{n} \psi_{j}+\frac{\pi}{2}-\epsilon, \sum_{j=1}^{n} \psi_{j}+\frac{\pi}{2}+\epsilon\right)$, an open interval of width 
$2 \epsilon$, which can be made arbitrarily small. Thus these self-expanders are almost calibrated, and Maslov zero.

The 'Lawlor necks' [15] have been used as local models in resolving intersection points of special Lagrangians; see for example, Butscher [5], Joyce [13], Dan Lee [16], and Yng-Ing Lee [17]. We expect the Lagrangian self-expanders found here will also play an important role in surgeries during Lagrangian mean curvature flow.

3.3. Other self-similar solutions from Theorems A, B. We now discuss the remaining solutions from Theorems A and B. As in Remark 3.2, without loss of generality we may take $\lambda_{j}= \pm 1, C=1$ and $A>0$. Section 3.2 dealt with the case $\lambda_{1}=\cdots=\lambda_{n}=1$ and $\alpha \geqslant 0$. There remain the cases (a) $\lambda_{1}=\cdots=\lambda_{n}=1$ and $\alpha<0$, and (b) at least one $\lambda_{j}$ is -1 . In (b), we reorder $w_{1}, \ldots, w_{n}$ if necessary so that $\lambda_{1}=\cdots=\lambda_{m}=1$ and $\lambda_{m+1}=\cdots=\lambda_{n}=-1$. We exclude $m=n$, as this is covered by $\S 3.2$ and case (a), and we exclude $m=0$, as then $L=\emptyset$. So we may take $1 \leqslant m<n$, and the following theorem covers all the remaining cases.

Theorem E. In Theorems $A$ and B, suppose that either:

(a) $\lambda_{1}=\cdots=\lambda_{n}=C=1, \alpha<0$ and $A>0$; or

(b) $\lambda_{1}=\cdots=\lambda_{m}=1$ and $\lambda_{m+1}=\cdots=\lambda_{n}=-1$ for some $1 \leqslant m<$ $n, C=1, A>0$, and $\alpha \in \mathbb{R}$.

Then solutions exist for all $s \in \mathbb{R}$, and we take $I=\mathbb{R}$. In each of cases (a), (b) we divide into two subcases:

(i) $\sum_{j=1}^{n} \frac{\lambda_{j}}{\alpha_{j}}+\alpha=0$ and $\alpha_{1} \cdots \alpha_{n}=A^{2}$; or

(ii) otherwise.

In case (i), we have explicit solutions to (5) and obtain

$$
\begin{gathered}
L=\left\{\left(x_{1} \sqrt{\alpha_{1}} e^{i\left(\psi_{1}-\lambda_{1} A s / \alpha_{1}\right)}, \ldots, x_{n} \sqrt{\alpha_{n}} e^{i\left(\psi_{n}-\lambda_{n} A s / \alpha_{n}\right)}\right):\right. \\
\left.x_{1}, \ldots, x_{n} \in \mathbb{R}, s \in \mathbb{R}, \sum_{j=1}^{n} \lambda_{j} x_{j}^{2}=1\right\},
\end{gathered}
$$

which is Hamiltonian stationary in addition to being self-similar, and invariant under a subgroup $\mathbb{R}$ or $\mathrm{U}(1)$ of diagonal matrices $\left\{\operatorname{diag}\left(e^{i \lambda_{1} t / \alpha_{1}}\right.\right.$, $\left.\left.\ldots, e^{i \lambda_{n} t / \alpha_{n}}\right): t \in \mathbb{R}\right\}$ in $\mathrm{U}(n)$.

In case (ii), $u$ and $\phi-\theta$ are periodic in $s$ with period $S>0$, and

$$
\begin{aligned}
u(s+S) & =u(s), & \phi_{j}(s+S) & =\phi_{j}(s)+\gamma_{j}, \\
\phi(s+S) & =\phi(s)+\sum_{j=1}^{n} \gamma_{j}, & \theta(s+S) & =\theta(s)+\sum_{j=1}^{n} \gamma_{j},
\end{aligned}
$$

for some $\gamma_{1}, \ldots, \gamma_{n} \in \mathbb{R}$ and all $s \in \mathbb{R}$. In case (b) with $\alpha=0$ we have $\theta(s) \equiv \theta(0)$ and $\sum_{j=1}^{n} \gamma_{j}=0$.

The Hamiltonian stationary self-similar solutions in (10) were obtained and studied by Lee and Wang in [19]. If we require the Lagrangian self-similar solutions in (6) to be Hamiltonian stationary, then they must be of the form (10) by some simple arguments. 
The solutions $w_{j}$ obtained in Theorem $\mathrm{E}$ are bounded and periodic or quasi-periodic. The periodic ones are much more interesting, as then $L$ is compact in case (a), and closed in case (b). Our next result explores this periodicity, and shows there are many periodic solutions.

Theorem F. In Theorem E, we say that $\left(w_{1}, \ldots, w_{n}\right)$ is periodic if there exists $T>0$ with $w_{j}(s)=w_{j}(s+T)$ for all $s \in \mathbb{R}$ and $j=1, \ldots, n$.

If $\left(w_{1}, \ldots, w_{n}\right)$ is periodic then in case (a), $L$ is a compact, immersed Lagrangian self-shrinker diffeomorphic to $\mathcal{S}^{1} \times \mathcal{S}^{n-1}$, and in case (b), $L$ is a closed, noncompact, immersed Lagrangian diffeomorphic to $\mathcal{S}^{1} \times$ $\mathcal{S}^{m-1} \times \mathbb{R}^{n-m}$, a self-expander if $\alpha>0$, a self-shrinker if $\alpha<0$, and special Lagrangian if $\alpha=0$.

In case $(\mathrm{i}),\left(w_{1}, \ldots, w_{n}\right)$ is periodic if and only if $\frac{\lambda_{j}}{\alpha_{j}}=\mu q_{j}$ with $\mu>0$ and $q_{j} \in \mathbb{Q}$ for $j=1, \ldots, n$. In case (ii), $\left(w_{1}, \ldots, w_{n}\right)$ is periodic if and only if $\gamma_{j} \in \pi \mathbb{Q}$ for $j=1, \ldots, n$. In both cases, for fixed $m, \alpha$, there is a dense subset of initial data for which $\left(w_{1}, \ldots, w_{n}\right)$ is periodic.

If $\left(w_{1}, \ldots, w_{n}\right)$ is not periodic, then $L$ is a noncompact, immersed Lagrangian diffeomorphic to $\mathcal{S}^{n-1} \times \mathbb{R}$ in case (a) and to $\mathcal{S}^{m-1} \times \mathbb{R}^{n-m+1}$ in case (b). It is not closed in $\mathbb{C}^{n}$, and the closure $\bar{L}$ of $L$ in $\mathbb{C}^{n}$ has dimension greater than $n$.

One can use the solutions obtained in Theorem $\mathrm{F}$ to form eternal solutions of Brakke flow without mass loss, which generalize some of Lee and Wang's earlier results $[\mathbf{1 8}, \mathbf{1 9}]$. Recall that Brakke flow $[\mathbf{3}]$ is a generalization of mean curvature flow to varifolds, measure-theoretic generalizations of submanifolds which may be singular. And an eternal solution is a solution which is defined for all $t$.

For $t \in \mathbb{R}$, define

$$
\begin{aligned}
L_{t}= & \left\{\left(x_{1} \sqrt{\alpha_{1}+u(s)} e^{i \phi_{1}(s)}, \ldots, x_{m} \sqrt{\alpha_{m}+u(s)} e^{i \phi_{m}(s)},\right.\right. \\
& \left.x_{m+1} \sqrt{\alpha_{m+1}-u(s)} e^{i \phi_{m+1}(s)}, \ldots, x_{n} \sqrt{\alpha_{n}-u(s)} e^{i \phi_{n}(s)}\right): \\
& \left.x_{1}, \ldots, x_{n} \in \mathbb{R}, s \in \mathbb{R} / T \mathbb{Z}, x_{1}^{2}+\cdots+x_{m}^{2}-x_{m+1}^{2}-\cdots-x_{n}^{2}=t\right\},
\end{aligned}
$$

where $u, \phi_{1}, \ldots, \phi_{n}$ are periodic with period $T>0$. Then $L_{t}$ is a closed, nonsingular, immersed Lagrangian self-expander in $\mathbb{C}^{n}$ diffeomorphic to $\mathcal{S}^{1} \times \mathcal{S}^{m-1} \times \mathbb{R}^{n-m}$ when $t>0$, and a closed, nonsingular, immersed Lagrangian self-shrinker in $\mathbb{C}^{n}$ diffeomorphic to $\mathcal{S}^{1} \times \mathcal{S}^{n-m-1} \times \mathbb{R}^{m}$ when $t<0$, and $L_{0}$ is a closed, immersed Lagrangian cone in $\mathbb{C}^{n}$ with link $\mathcal{S}^{1} \times \mathcal{S}^{m-1} \times \mathcal{S}^{n-m-1}$, with an isolated singular point at 0 .

The fact that $L_{t}$ form an eternal solution of Brakke flow without mass loss is proved in Lee and Wang's paper [19].

3.4. Translating solutions. In $\S 3.1-\S 3.3$ we have considered only centered quadrics centered at 0, and only Lagrangian self-expanders and self-shrinkers. It is an obvious question whether we can generalize the 
constructions to non-centered quadrics on the one hand, and to Lagrangian translating solitons on the other. In fact it seems to be natural to put these ideas together, and to construct Lagrangian translating solitons using non-centered quadrics whose favoured axis is the direction of translation. Here is the class of $n$-submanifolds of $\mathbb{C}^{n}$ amongst which we will seek Lagrangian translating solitons.

Ansatz 3.3. Fix $n \geqslant 2$. Consider $n$-submanifolds $L$ in $\mathbb{C}^{n}$ of the form:

$$
\begin{aligned}
& L=\left\{\left(x_{1} w_{1}(s), \ldots, x_{n-1} w_{n-1}(s), x_{n}+\beta(s)\right):\right. \\
& \left.\quad s \in I, x_{1}, \ldots, x_{n} \in \mathbb{R}, \sum_{j=1}^{n-1} \lambda_{j} x_{j}^{2}+2 x_{n}=0\right\},
\end{aligned}
$$

where $\lambda_{1}, \ldots, \lambda_{n-1} \in \mathbb{R} \backslash\{0\}$ are nonzero constants, $I$ is an open interval in $\mathbb{R}$, and $w_{1}, \ldots, w_{n-1}: I \rightarrow \mathbb{C} \backslash\{0\}, \beta: I \rightarrow \mathbb{C}$ are smooth functions. We want $L$ to satisfy:

(i) $L$ is Lagrangian;

(ii) the Lagrangian angle $\theta: L \rightarrow \mathbb{R}$ or $\theta: L \rightarrow \mathbb{R} / 2 \pi \mathbb{Z}$ of $L$ is a function only of $s$, not of $x_{1}, \ldots, x_{n}$; and

(iii) $L$ is a translating soliton under mean curvature flow in $\mathbb{C}^{n}$, with translating vector $(0, \ldots, 0, \alpha) \in \mathbb{C}^{n}$, for $\alpha \in \mathbb{R}$.

One motivation for this is the special Lagrangian submanifolds found by the first author [12, §7], which are of the form (11). Another is the limiting argument used to prove Theorem $\mathrm{G}$ below, which recovers Ansatz 3.3 as a limit of Ansatz 3.1. Here is the analogue of Theorem A for this ansatz.

Theorem G. Let $\lambda_{1}, \ldots, \lambda_{n-1} \in \mathbb{R} \backslash\{0\}$ and $\alpha \in \mathbb{R}$ be constants, $I$ be an open interval in $\mathbb{R}$, and $\theta: I \rightarrow \mathbb{R}$ or $\theta: I \rightarrow \mathbb{R} / 2 \pi \mathbb{Z}, w_{1}, \ldots, w_{n-1}$ : $I \rightarrow \mathbb{C} \backslash\{0\}$ and $\beta: I \rightarrow \mathbb{C}$ be smooth functions. Suppose that

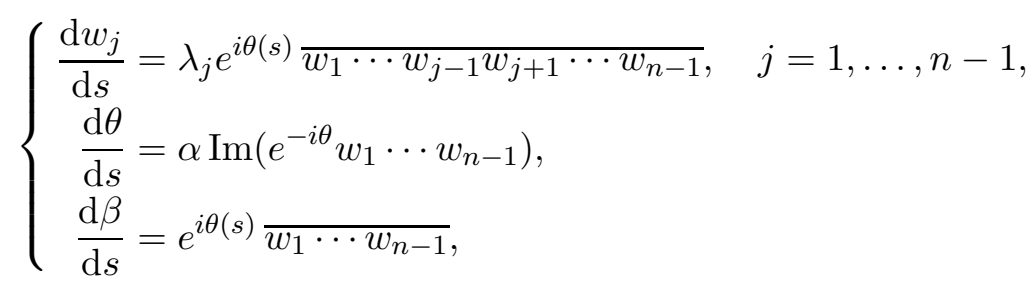

hold in $I$. Then the submanifold $L$ in $\mathbb{C}^{n}$ given by

$$
\begin{array}{r}
L=\left\{\left(x_{1} w_{1}(s), \ldots, x_{n-1} w_{n-1}(s),-\frac{1}{2} \sum_{j=1}^{n-1} \lambda_{j} x_{j}^{2}+\beta(s)\right):\right. \\
\left.x_{1}, \ldots, x_{n-1} \in \mathbb{R}, \quad s \in I\right\}
\end{array}
$$

is an embedded Lagrangian diffeomorphic to $\mathbb{R}^{n}$, with Lagrangian angle $\theta(s)$. When $\alpha \neq 0$, it is a Lagrangian translating soliton with translating vector $(0, \ldots, 0, \alpha) \in \mathbb{C}^{n}$. When $\alpha=0$, it is special Lagrangian, and the construction reduces to that of Joyce $[\mathbf{1 2}, \S 7]$. 
We can prove this directly, following the proof of Theorem A in $\S 4$. This is straightforward, and we leave it as an exercise for the interested reader. Instead, we give a somewhat informal proof which obtains Theorem $\mathrm{G}$ from Theorem A by a limiting procedure, since this gives more insight into why the construction should generalize in this way.

Proof of Theorem $G$, assuming Theorem $A$. Let $\lambda_{1}, \ldots, \lambda_{n-1} \in \mathbb{R} \backslash\{0\}$ and $\alpha \in \mathbb{R}$ be constants, $I$ be an open interval in $\mathbb{R}$, and $\theta: I \rightarrow \mathbb{R}$ or $\theta: I \rightarrow \mathbb{R} / 2 \pi \mathbb{Z}, w_{1}, \ldots, w_{n-1}: I \rightarrow \mathbb{C} \backslash\{0\}$ and $\beta: I \rightarrow \mathbb{C}$ be smooth functions. Let $R>0$. Define constants $\tilde{\lambda}_{1}, \ldots, \tilde{\lambda}_{n}, \tilde{C} \in \mathbb{R} \backslash\{0\}$ and $\tilde{\alpha} \in \mathbb{R}$, an open interval $\tilde{I}$, and smooth $\tilde{\theta}: \tilde{I} \rightarrow \mathbb{R}$ or $\tilde{\theta}: \tilde{I} \rightarrow \mathbb{R} / 2 \pi \mathbb{Z}$, $\tilde{w}_{1}, \ldots, \tilde{w}_{n}: \tilde{I} \rightarrow \mathbb{C} \backslash\{0\}$ by

$$
\begin{gathered}
\tilde{\lambda}_{j}=\lambda_{j}, \quad j=1, \ldots, n-1, \quad \tilde{\lambda}_{n}=R, \tilde{C}=R, \quad \tilde{\alpha}=\alpha, \tilde{I}=R^{-1} I, \\
\tilde{s}=R^{-1} s, \quad \tilde{w}_{j}(\tilde{s})=w_{j}(R \tilde{s})=w_{j}(s), j=1, \ldots, n-1, \\
\tilde{w}_{n}(\tilde{s})=R+\beta(R \tilde{s})=R+\beta(s), \tilde{\theta}(\tilde{s})=\theta(R \tilde{s})=\theta(s) .
\end{gathered}
$$

We suppose that $\beta \neq-R$ so that $\tilde{w}_{n}$ maps $\tilde{I} \rightarrow \mathbb{C} \backslash\{0\}$.

Apply Theorem A to this new data $\tilde{\lambda}_{j}, \tilde{C}, \tilde{\alpha}, \tilde{I}, \tilde{\theta}, \tilde{w}_{j}$. This yields o.d.e.s (3) upon $\tilde{w}_{j}, \tilde{\theta}$, in terms of derivatives with respect to $\tilde{s}$, and defines (4) a self-similar Lagrangian $\tilde{L}$ in $\mathbb{C}^{n}$ when these o.d.e.s hold. Define $L=\tilde{L}-(0, \ldots, 0, R)$, that is, $L$ is $\tilde{L}$ translated by the vector $-(0, \ldots, 0, R)$. Rewriting the o.d.e.s (3) in terms of $R, \lambda_{j}, \alpha, I, \theta, w_{j}, \beta$ using (14) yields

$$
\left\{\begin{array}{l}
\frac{\mathrm{d} w_{j}}{\mathrm{~d} s}=\lambda_{j} e^{i \theta(s)} \overline{w_{1} \cdots w_{j-1} w_{j+1} \cdots w_{n-1}}\left(1+R^{-1} \bar{\beta}(s)\right), \text { all } j, \\
\frac{\mathrm{d} \theta}{\mathrm{d} s}=\alpha \operatorname{Im}\left(e^{-i \theta} w_{1} \cdots w_{n-1}\left(1+R^{-1} \beta(s)\right)\right), \\
\frac{\mathrm{d} \beta}{\mathrm{d} s}=e^{i \theta(s)} \overline{w_{1} \cdots w_{n-1}} .
\end{array}\right.
$$

Rewriting $\tilde{L}$ in (4) in terms of $R, \lambda_{j}, \alpha, I, \theta, w_{j}, \beta$, translating by - $(0$, $\ldots, 0, R)$ to get $L$, and replacing $x_{n}$ in (4) by $1+R^{-1} \bar{x}_{n}$, yields

$$
\begin{aligned}
L=\{ & \left(\left(x_{1} w_{1}(s), \ldots, x_{n-1} w_{n-1}(s), \bar{x}_{n}+\beta(s)+R^{-1} \beta(s) \bar{x}_{n}\right):\right. \\
& \left.x_{1}, \ldots, x_{n-1}, \bar{x}_{n} \in \mathbb{R}, s \in I, \sum_{j=1}^{n-1} \lambda_{j} x_{j}^{2}+2 \bar{x}_{n}+R^{-1} \bar{x}_{n}^{2}=0\right\} .
\end{aligned}
$$

The conclusion of Theorem A is that $\tilde{L}$ satisfies $\alpha F^{\perp}=R H$. Since $L$ is the translation of $\tilde{L}$ by $-(0, \ldots, 0, R)$, and this subtracts $(0, \ldots, 0, R)$ from $F$, we see that $L$ satisfies $\alpha(F+(0, \ldots, 0, R))^{\perp}=R H$. Dividing by $R$ and setting $T=(0, \ldots, 0, \alpha)$, this shows that $L$ satisfies $H=$ $T^{\perp}+R^{-1} \alpha F^{\perp}$.

Now let us take the limit $R \rightarrow \infty$. Then (15) reduces to (12), as the $R^{-1}$ terms disappear, and (16) reduces to (13), as $\bar{x}_{n}=-\frac{1}{2} \sum_{j=1}^{n-1} \lambda_{j} x_{j}^{2}$ in the limit. The equation $H=T^{\perp}+R^{-1} \alpha F^{\perp}$ for $L$ becomes $H=T^{\perp}$, so $L$ is a Lagrangian translating soliton with translating vector $T=$ $(0, \ldots, 0, \alpha)$. 
It remains to show that $L$ is embedded, that is, the immersion $\iota$ : $\left(x_{1}, \ldots, x_{n-1}, s\right) \mapsto\left(x_{1} w_{1}(s), \ldots, x_{n-1} w_{n-1}(s),-\frac{1}{2} \sum_{j=1}^{n-1} \lambda_{j} x_{j}^{2}+\beta(s)\right)$ is injective. Combining (15) with equation (7) of Theorem $\mathrm{B}$ gives $\operatorname{Im} \frac{\mathrm{d} \beta}{\mathrm{d} s} \equiv-A e^{-\alpha u / 2}$ for some $A \in \mathbb{R}$. Thus $\operatorname{Im} \beta$ is strictly decreasing in $s$ if $A>0$, and strictly increasing if $A<0$. In both cases, if $\iota\left(x_{1}, \ldots, x_{n-1}, s\right)=\left(z_{1}, \ldots, z_{n}\right)$ then $\operatorname{Im} z_{n}$ determines $s$, and given $s$, we have $x_{j}=w_{j}(s)^{-1} z_{j}$ for $j=1, \ldots, n-1$. So $\iota$ is injective if $A \neq 0$. When $A=0$ we can solve explicitly and show $\iota$ is injective. q.e.d.

Note that the first two equations in (12) are exactly the same as (3), replacing $n$ by $n-1$. Having chosen some solutions $w_{1}, \ldots, w_{n-1}, \theta$ to the first two equations of (12), the third equation of (12) determines $\beta$ uniquely up to $\beta \mapsto \beta+c$, by integration. Actually we can write $\beta$ explicitly in terms of $u, \theta$ : in the notation of Theorem $\mathrm{B}$, if $\alpha \neq 0$ then (5) and the last equation of (12) give $\frac{\mathrm{d} \beta}{\mathrm{d} s}=\frac{1}{2} \frac{\mathrm{d} u}{\mathrm{~d} s}-\frac{i}{\alpha} \frac{\mathrm{d} \theta}{\mathrm{d} s}$, which integrates to $\beta(s)=\frac{1}{2} u(s)-\frac{i}{\alpha} \theta(s)+K$, for $K \in \mathbb{C}$. So we deduce:

Corollary H. In the situation of Theorem $G$, when $\alpha \neq 0$, the Lagrangian translating soliton $L$ may be rewritten

$$
\begin{array}{r}
L=\left\{\left(x_{1} \sqrt{\alpha_{1}+\lambda_{1} u(s)} e^{i \phi_{1}(s)}, \ldots, x_{n-1} \sqrt{\alpha_{n-1}+\lambda_{n-1} u(s)} e^{i \phi_{n-1}(s)},\right.\right. \\
\left.\left.\quad \frac{1}{2} u(s)-\frac{1}{2} \sum_{j=1}^{n-1} \lambda_{j} x_{j}^{2}-\frac{i}{\alpha} \theta(s)+K\right): x_{1}, \ldots, x_{n-1} \in \mathbb{R}, \quad s \in I\right\},
\end{array}
$$

where $K \in \mathbb{C}$ and $u, \alpha_{1}, \ldots, \alpha_{n-1}, \phi_{1}, \ldots, \phi_{n-1}$ are as in Theorem $B$ with $n-1$ in place of $n$, and satisfy (5) and (7) for some $A \in \mathbb{R}$.

Proposition 2.5 implies that the Lagrangian angle $\theta$ of $L$ should be of the form $-\left.\alpha \operatorname{Im} z_{n}\right|_{L}+c$, where $\left(z_{1}, \ldots, z_{n}\right)$ are the complex coordinates on $\mathbb{C}^{n}$. The imaginary part of the last coordinate in $(17)$ is $-\frac{1}{\alpha} \theta(s)+$ $\operatorname{Im} K$, so the proposition holds with $c=\alpha \operatorname{Im} K$.

Theorems C, D, E, and F give a good description of solutions of (5), and hence of the Lagrangian translating solitons $L$ from Theorem $G$ and Corollary H. In the authors' opinion, the most interesting case of Theorem $\mathrm{G}$ is when $\lambda_{1}, \ldots, \lambda_{n-1}>0$ and $\alpha \geqslant 0$. (This is equivalent to the case $\lambda_{1}, \ldots, \lambda_{n-1}<0$ and $\alpha \leqslant 0$, changing the sign of the last coordinate in $\mathbb{C}^{n}$.) The following result combines Theorems $\mathrm{C}$, D with $n-1$ in place of $n$, Theorem $\mathrm{G}$, and Corollary H. For simplicity we set $\psi_{1}=\cdots=\psi_{n-1}=0$ and $K=-\frac{1}{2} u_{*}$, where $u_{*}$ is defined in the proof of Theorem C (see Figure 1 on page 147).

Corollary I. For given constants $\alpha \geqslant 0$ and $a_{1}, \ldots, a_{n-1}>0$, define

$\phi_{j}(y)=\int_{0}^{y} \frac{\mathrm{d} t}{\left(\frac{1}{a_{j}}+t^{2}\right) \sqrt{P(t)}}$, where $P(t)=\frac{1}{t^{2}}\left(\prod_{k=1}^{n-1}\left(1+a_{k} t^{2}\right) e^{\alpha t^{2}}-1\right)$, 
for $j=1, \ldots, n-1$ and $y \in \mathbb{R}$. Then when $\alpha \neq 0$,

$L=\left\{\left(x_{1} \sqrt{\frac{1}{a_{1}}+y^{2}} e^{i \phi_{1}(y)}, \ldots, x_{n-1} \sqrt{\frac{1}{a_{n-1}}+y^{2}} e^{i \phi_{n-1}(y)}, \frac{1}{2} y^{2}-\frac{1}{2} \sum_{j=1}^{n-1} x_{j}^{2}\right.\right.$

$$
\left.\left.-\frac{i}{\alpha} \sum_{j=1}^{n-1} \phi_{j}(y)-\frac{i}{\alpha} \arg \left(y+i P(y)^{-1 / 2}\right)\right): x_{1}, \ldots, x_{n-1}, y \in \mathbb{R}\right\}
$$

is a closed, embedded Lagrangian in $\mathbb{C}^{n}$ diffeomorphic to $\mathbb{R}^{n}$, which is a Lagrangian translating soliton with translating vector $(0, \ldots, 0, \alpha) \in \mathbb{C}^{n}$. When $\alpha=0$,

$$
\begin{aligned}
& L=\{\left(x_{1} \sqrt{\frac{1}{a_{1}}+y^{2}} e^{i \phi_{1}(y)}, \ldots, x_{n-1} \sqrt{\frac{1}{a_{n-1}}+y^{2}} e^{i \phi_{n-1}(y)}, \frac{1}{2} y^{2}-\frac{1}{2} \sum_{j=1}^{n-1} x_{j}^{2}\right. \\
&\left.\left.+i \int_{0}^{y} \frac{\mathrm{d} t}{\sqrt{\frac{1}{t^{2}}\left(\prod_{k=1}^{n}\left(1+a_{k} t^{2}\right)-1\right)}}\right): x_{1}, \ldots, x_{n-1}, y \in \mathbb{R}\right\}
\end{aligned}
$$

is a closed, embedded Lagrangian in $\mathbb{C}^{n}$ diffeomorphic to $\mathbb{R}^{n}$, which is special Lagrangian.

There exist $\bar{\phi}_{1}, \ldots, \bar{\phi}_{n-1} \in\left(0, \frac{\pi}{2}\right]$ such that $\phi_{j}(y) \rightarrow \bar{\phi}_{j}$ as $y \rightarrow \infty$ and $\phi_{j}(y) \rightarrow-\bar{\phi}_{j}$ as $y \rightarrow-\infty$ for $j=1, \ldots, n-1$. These satisfy $\bar{\phi}_{1}+\cdots+\bar{\phi}_{n-1}<\frac{\pi}{2}$ if $\alpha>0$, and $\bar{\phi}_{1}+\cdots+\bar{\phi}_{n-1}=\frac{\pi}{2}$ if $\alpha=0$. For fixed $\alpha>0$, the map $\left(a_{1}, \ldots, a_{n-1}\right) \mapsto\left(\bar{\phi}_{1}, \ldots, \bar{\phi}_{n-1}\right)$ is a $1-1$ correspondence from $(0, \infty)^{n-1}$ to $\left\{\left(\bar{\phi}_{1}, \ldots, \bar{\phi}_{n-1}\right) \in\left(0, \frac{\pi}{2}\right)^{n-1}: \bar{\phi}_{1}+\cdots+\bar{\phi}_{n-1}<\frac{\pi}{2}\right\}$. When $\alpha=0$, the map has image $\left\{\left(\bar{\phi}_{1}, \ldots, \bar{\phi}_{n-1}\right) \in\left(0, \frac{\pi}{2}\right]^{n-1}: \bar{\phi}_{1}+\cdots+\right.$ $\left.\bar{\phi}_{n-1}=\frac{\pi}{2}\right\}$.

The Lagrangian angle of $L$ in (18) varies between $\sum_{j=1}^{n-1} \bar{\phi}_{j}$ and $\pi-$ $\sum_{j=1}^{n-1} \bar{\phi}_{j}$. Thus, when $\alpha>0$, by choosing $\sum_{j=1}^{n-1} \bar{\phi}_{j}$ close to $\frac{\pi}{2}$, the oscillation of the Lagrangian angle of $L$ can be made arbitrarily small.

We can give the following heuristic description of $L$ in (18). If $y \gg 0$ then $\phi_{j}(y) \approx \bar{\phi}_{j}$ and $\sqrt{\frac{1}{a_{j}}+y^{2}} \approx y$, and the terms $-\frac{i}{\alpha} \sum_{j=1}^{n} \phi_{j}(y)-$ $\frac{i}{\alpha} \arg \left(y+i P(y)^{-1 / 2}\right)$ are negligible compared to $\frac{1}{2} y^{2}$ in the last coordinate. Thus, the region of $L$ with $y \gg 0$ is in a weak sense approximate to

$$
\left\{\left(x_{1} y e^{i \bar{\phi}_{1}}, \ldots, x_{n-1} y e^{i \bar{\phi}_{n-1}}, \frac{1}{2} y^{2}-\frac{1}{2} \sum_{j=1}^{n-1} x_{j}^{2}\right): x_{1}, \ldots, x_{n-1} \in \mathbb{R}, y>0\right\} \text {. }
$$

But this is just an unusual way of parametrizing

$$
L_{1}=\left\{\left(y_{1} e^{i \bar{\phi}_{1}}, \ldots, y_{n-1} e^{i \bar{\phi}_{n-1}}, y_{n}\right): y_{j} \in \mathbb{R}\right\} \backslash\left\{\left(0, \ldots, 0, y_{n}\right): y_{n} \leqslant 0\right\},
$$

the complement of a ray in a Lagrangian plane. Similarly, the region of $L$ with $y \ll 0$ is in a weak sense approximate to

$$
L_{2}=\left\{\left(y_{1} e^{-i \bar{\phi}_{1}}, \ldots, y_{n-1} e^{-i \bar{\phi}_{n-1}}, y_{n}\right): y_{j} \in \mathbb{R}\right\} \backslash\left\{\left(0, \ldots, 0, y_{n}\right): y_{n} \leqslant 0\right\} \text {. }
$$

So, $L$ can be roughly described as asymptotic to the union of two Lagrangian planes $L_{1}, L_{2} \cong \mathbb{R}^{n}$ which intersect in an $\mathbb{R}$ in $\mathbb{C}^{n}$, the $y_{n}$-axis $\left\{\left(0, \ldots, 0, y_{n}\right): y_{n} \in \mathbb{R}\right\}$. To make $L$, we glue these Lagrangian planes 
by a kind of 'connect sum' along the negative $y_{n}$-axis $\left\{\left(0, \ldots, 0, y_{n}\right)\right.$ : $\left.y_{n} \leqslant 0\right\}$. Under Lagrangian mean curvature flow, $L_{1}, L_{2}$ remain fixed, but the gluing region translates in the positive $y_{n}$ direction, as though $L_{1}, L_{2}$ are being 'zipped together'.

Note too that when the oscillation of the Lagrangian angle of $L$ is small compared to $|\alpha|$, we see from Proposition 2.5 that $\left.\operatorname{Im} z_{n}\right|_{L}$ is confined to a small interval, where $\left(z_{1}, \ldots, z_{n}\right)$ are the complex coordinates on $\mathbb{C}^{n}$. That is, $L$ is close to the affine $\mathbb{R}^{2 n-1}$ in $\mathbb{C}^{n}$ defined by $\operatorname{Im} z_{n}=-\frac{\pi}{2 \alpha}$.

Question 3.4. Can the translating solitons with small Lagrangian angle oscillation in Corollary I arise as blow-ups of finite time singularities for Lagrangian mean curvature flow, particularly when $n=2$ ?

It is important to answer this question in developing a regularity theory for the flow. Such relations have been observed before in codimension one mean curvature by White $[\mathbf{2 8}, \mathbf{2 9}]$, and Huisken and Sinestrari [11], and in Ricci flow by Perelman [22]. See also the recent work by Neves and Tian $[\mathbf{2 1}]$ for related discussions.

We can also ask about the Lagrangian translating solitons from Theorem $\mathrm{G}$ and Corollary $\mathrm{H}$ coming from Theorem $\mathrm{E}$ with $n-1$ in place of $n$. As in Theorem E, we take $I=\mathbb{R}$. Using the notation of Theorem B for $w_{1}, \ldots, w_{n-1}, \theta$, observe that the third equation of (12) gives

$$
\operatorname{Im} \frac{\mathrm{d} \beta}{\mathrm{d} s}=-Q(u)^{1 / 2} \sin (\phi-\theta)=-A e^{-\alpha u / 2} .
$$

As in Remark 3.2(d), when $A=0$ the Lagrangian $L$ is an open subset of an affine Lagrangian plane $\mathbb{R}^{n}$ in $\mathbb{C}^{n}$, which is not interesting, so we restrict to $A \neq 0$. Then (19) shows that either $\operatorname{Im} \frac{\mathrm{d} \beta}{\mathrm{d} s}>0$ for all $s \in \mathbb{R}$, or $\operatorname{Im} \frac{\mathrm{d} \beta}{\mathrm{d} s}<0$ for all $s \in \mathbb{R}$. Thus $\beta$ can never be periodic, so we have no analogue of Theorem $\mathrm{F}$ in the translating case. We can also deduce from this that the Lagrangians are closed, embedded, diffeomorphic to $\mathbb{R}^{n}$, and when $\alpha \neq 0$ have infinite oscillation of the Lagrangian angle. This implies that these Lagrangian translating solitons from Theorems $\mathrm{G}$ and $\mathrm{E}$ cannot arise as blow-ups of finite time singularities for Lagrangian mean curvature flow.

\section{A construction for self-similar Lagrangians}

We now prove:

Theorem A. Let $\lambda_{1}, \ldots, \lambda_{n}, C \in \mathbb{R} \backslash\{0\}$ and $\alpha \in \mathbb{R}$ be constants, $I$ be an open interval in $\mathbb{R}$, and $\theta: I \rightarrow \mathbb{R}$ or $\theta: I \rightarrow \mathbb{R} / 2 \pi \mathbb{Z}$ and 
$w_{1}, \ldots, w_{n}: I \rightarrow \mathbb{C} \backslash\{0\}$ be smooth functions. Suppose that

$$
\begin{aligned}
\frac{\mathrm{d} w_{j}}{\mathrm{~d} s} & =\lambda_{j} e^{i \theta(s)} \overline{w_{1} \cdots w_{j-1} w_{j+1} \cdots w_{n}}, \quad j=1, \ldots, n, \\
\frac{\mathrm{d} \theta}{\mathrm{d} s} & =\alpha \operatorname{Im}\left(e^{-i \theta(s)} w_{1} \cdots w_{n}\right),
\end{aligned}
$$

hold in I. Then the submanifold $L$ in $\mathbb{C}^{n}$ given by

$$
L=\left\{\left(x_{1} w_{1}(s), \ldots, x_{n} w_{n}(s)\right): s \in I, x_{j} \in \mathbb{R}, \sum_{j=1}^{n} \lambda_{j} x_{j}^{2}=C\right\},
$$

is Lagrangian, with Lagrangian angle $\theta(s)$ at $\left(x_{1} w_{1}(s), \ldots, x_{n} w_{n}(s)\right)$, and its position vector $F$ and mean curvature vector $H$ satisfy $\alpha F^{\perp}=$ $C H$. That is, $L$ is a self-expander when $\alpha / C>0$ and a self-shrinker when $\alpha / C<0$. When $\alpha=0$ the Lagrangian angle $\theta$ is constant, so that $L$ is special Lagrangian, with $H=0$. In this case the construction reduces to that of Joyce $[\mathbf{1 2}, \S 5]$.

Proof. Define $\Sigma=\left\{\left(x_{1}, \ldots, x_{n}\right) \in \mathbb{R}^{n}: \sum_{j=1}^{n} \lambda_{j} x_{j}^{2}=C\right\}$. Then $\Sigma$ is a nonsingular quadric in $\mathbb{R}^{n}$, an $(n-1)$-manifold. Define a smooth $\operatorname{map} \iota: \Sigma \times I \rightarrow \mathbb{C}^{n}$ by $\iota:\left(\left(x_{1}, \ldots, x_{n}\right), s\right) \longmapsto\left(x_{1} w_{1}(s), \ldots, x_{n} w_{n}(s)\right)$. Then $L=\iota(\Sigma \times I)$. The determinant calculation below implies $\iota$ is an immersion, and so $L$ is a nonsingular immersed $n$-submanifold in $\mathbb{C}^{n}$.

Fix $\boldsymbol{x}=\left(x_{1}, \ldots, x_{n}\right) \in \Sigma$ and $s \in I$. We will find the tangent space $T_{\iota(\boldsymbol{x}, s)} L$, show that it is Lagrangian, and compute its Lagrangian angle. Let $e_{1}, \ldots, e_{n-1}$ be an orthonormal basis for $T_{\boldsymbol{x}} \Sigma$ in $\mathbb{R}^{n}$, and write $e_{j}=\left(a_{j 1}, \ldots, a_{j n}\right)$ in $\mathbb{R}^{n}$ for $j=1, \ldots, n-1$. Let $e_{n}=\left(\sum_{j=1}^{n} \lambda_{j}^{2} x_{j}^{2}\right)^{-1 / 2}$. $\left(\lambda_{1} x_{1}, \ldots, \lambda_{n} x_{n}\right)$. Then $e_{n}$ is a unit normal vector to $\Sigma$ at $\boldsymbol{x}$ in $\mathbb{R}^{n}$. Let $e_{1}, \ldots, e_{n-1}$ be chosen so that $e_{1}, \ldots, e_{n-1}, e_{n}$ is an oriented orthonormal basis for $\mathbb{R}^{n}$. Then $\operatorname{det}\left(e_{1} \ldots e_{n}\right)=1$, regarding $e_{1}, \ldots, e_{n}$ as column vectors, and $\left(e_{1} \ldots e_{n}\right)$ as an $n \times n$ matrix.

Now $e_{1}, \ldots, e_{n-1}, \frac{\partial}{\partial s}$ is a basis for $T_{(\boldsymbol{x}, s)}(\Sigma \times I)$. Define $f_{j}=\mathrm{d} \iota\left(e_{j}\right) \in$ $\mathbb{C}^{n}$ for $j=1, \ldots, n-1$ and $f_{n}=\mathrm{d} \iota\left(\frac{\partial}{\partial s}\right) \in \mathbb{C}^{n}$. Then $f_{1}, \ldots, f_{n}$ is a basis for $T_{\iota(\boldsymbol{x}, s)} L$, over $\mathbb{R}$. From the definitions we have $f_{j}=$ $\left(a_{j 1} w_{1}(s), \ldots, a_{j n} w_{n}(s)\right)$ for $j<n$, and $f_{n}=\left(x_{1} \dot{w}_{1}(s), \ldots, x_{n} \dot{w}_{n}(s)\right)$. Therefore

$$
\begin{aligned}
& \left\langle f_{j}, J f_{k}\right\rangle=\operatorname{Re}\left(-i \sum_{l=1}^{n} a_{j l} a_{k l}\left|w_{l}\right|^{2}\right)=0, \quad \text { for } j, k=1, \ldots, n-1, \\
& \left\langle f_{j}, J f_{n}\right\rangle=\operatorname{Re}\left(-i w_{1} \cdots w_{n} e^{-i \theta} \sum_{j=1}^{n} a_{j l} \lambda_{l} x_{l}\right)=0, \text { for } j=1, \ldots, n-1,
\end{aligned}
$$

where in the second equation we use the first equation of (20) to substitute for $\dot{w}_{l}(s)$, and the fact that $\left(\lambda_{1} x_{1}, \ldots, \lambda_{n} x_{n}\right)$ is normal to $\Sigma$ at $\boldsymbol{x}$, and so orthogonal to $e_{j}=\left(a_{j 1}, \ldots, a_{j n}\right)$.

Thus $\left\langle f_{j}, J f_{k}\right\rangle=0$ for $j, k=1, \ldots, n$, so the symplectic form $\omega(*, *)=$ $\langle *, J *\rangle$ on $\mathbb{C}^{n}$ vanishes on $\left\langle f_{1}, \ldots, f_{n}\right\rangle_{\mathbb{R}}=T_{\iota(\boldsymbol{x}, s)} L$, and $T_{\iota(\boldsymbol{x}, s)} L$ is a Lagrangian plane in $\mathbb{C}^{n}$. Hence $L$ is Lagrangian. To compute the Lagrangian angle, write $w_{j}(s)=r_{j}(s) e^{i \phi_{j}(s)}$ and $\phi(s)=\sum_{j=1}^{n} \phi_{j}(s)$, where 


$$
\begin{aligned}
& r_{j}(s)=\left|w_{j}(s)\right| \text {. Then } \\
& \operatorname{det}\left(f_{1} \cdots f_{n}\right) \\
& =\left|\begin{array}{cccc}
a_{11} r_{1} e^{i \phi_{1}(s)} & \cdots & a_{(n-1) 1} r_{1} e^{i \phi_{1}(s)} & x_{1} \lambda_{1} r_{2} \cdots r_{n} e^{i\left(\phi_{1}(s)+\theta(s)-\phi(s)\right)} \\
a_{12} r_{2} e^{i \phi_{2}(s)} & \cdots & a_{(n-1) 2} r_{2} e^{i \phi_{2}(s)} & x_{2} \lambda_{2} r_{1} r_{3} \cdots r_{n} e^{i\left(\phi_{2}(s)+\theta(s)-\phi(s)\right)} \\
\vdots & \vdots & \vdots & \vdots \\
a_{1 n} r_{n} e^{i \phi_{n}(s)} & \cdots & a_{(n-1) n} r_{n} e^{i \phi_{n}(s)} & x_{n} \lambda_{n} r_{1} \cdots r_{n-1} e^{i\left(\phi_{n}(s)+\theta(s)-\phi(s)\right)}
\end{array}\right| \\
& =r_{1}^{2} \cdots r_{n}^{2} e^{i \theta}\left|\begin{array}{cccc}
a_{11} & \cdots & a_{(n-1) 1} & \frac{x_{1} \lambda_{1}}{r_{1}^{2}} \\
a_{12} & \cdots & a_{(n-1) 2} & \frac{x_{2} \lambda_{2}}{r_{2}^{2}} \\
\vdots & \vdots & \vdots & \vdots \\
a_{1 n} & \cdots & a_{(n-1) n} & \frac{x_{n} \lambda_{n}}{r_{n}^{2}}
\end{array}\right|=\frac{r_{1}^{2} \cdots r_{n}^{2} e^{i \theta}}{\sqrt{\sum_{l=1}^{n} \lambda_{l}^{2} x_{l}^{2}}} \sum_{l=1}^{n} \frac{\lambda_{l}^{2} x_{l}^{2}}{r_{l}^{2}} .
\end{aligned}
$$

Here in the second step we extract factors of $r_{j} e^{i \phi_{j}(s)}$ from the $j^{\text {th }}$ row for $j=1, \ldots, n$, and a factor $r_{1} \cdots r_{n} e^{i(\theta(s)-\phi(s))}$ from the $n^{\text {th }}$ column. The factors $e^{i \phi_{1}(s)} e^{i \phi_{2}(s)} \cdots e^{i \phi_{n}(s)} e^{-i \phi(s)}$ cancel to give 1. In the third and final step, we note that the first $n-1$ columns of the matrix on the third line are $e_{1}, \ldots, e_{n-1}$, and $e_{1}, \ldots, e_{n}$ are orthonormal with $\operatorname{det}\left(e_{1} \ldots e_{n}\right)=1$, so we project the vector $\left(\frac{\lambda_{1} x_{1}}{r_{1}^{2}}, \ldots, \frac{\lambda_{n} x_{n}}{r_{n}^{2}}\right)$ to $e_{n}$ to calculate the determinant. This shows that the Lagrangian angle on $L$ at $\left(x_{1} w_{1}(s), \ldots, x_{n} w_{n}(s)\right)$ is $\theta(s)$, as we have to prove. Also, as it shows that $\operatorname{det}\left(f_{1} \cdots f_{n}\right) \neq 0$, this calculation implies that $\mathrm{d} \iota: T_{(\boldsymbol{x}, s)}(\Sigma \times I) \rightarrow \mathbb{C}^{n}$ is injective, and $\iota$ is an immersion, as we claimed above.

The matrix $\left(g_{a b}\right)$ of the metric on $L$ w.r.t. the basis $f_{1}, \ldots, f_{n}$ is

$$
g_{n n}=r_{1}^{2} \cdots r_{n}^{2} \sum_{l=1}^{n} \frac{\lambda_{l}^{2} x_{l}^{2}}{r_{l}^{2}}, g_{j n}=g_{n j}=0, \text { and } g_{j k}=\sum_{l=1}^{n} a_{j l} a_{k l} r_{l}^{2}
$$

for $j, k=1, \ldots, n-1$. Hence by (20) the mean curvature vector is

$$
H=J \nabla \theta=\frac{\dot{\theta}}{g_{n n}} J f_{n}=\frac{\alpha r_{1} \cdots r_{n} \sin (\phi-\theta)}{g_{n n}} J f_{n} .
$$

The normal projection of the position vector $F$ is computed by

$$
\begin{aligned}
\left\langle F, J f_{l}\right\rangle & =\operatorname{Re}\left(-i \sum_{j=1}^{n} r_{j}^{2} x_{j} a_{l j}\right)=0, \\
\left\langle F, J f_{n}\right\rangle & =\operatorname{Re}\left(-i r_{1} \cdots r_{n} e^{i(\phi-\theta)} \sum_{j=1}^{n} \lambda_{j} x_{j}^{2}\right)=C r_{1} \cdots r_{n} \sin (\phi-\theta) .
\end{aligned}
$$

It follows that

$$
F^{\perp}=\frac{C r_{1} \cdots r_{n} \sin (\phi-\theta)}{g_{n n}} J f_{n} .
$$

Equations (23) and (24) give $\alpha F^{\perp}=C H$, as we have to prove. q.e.d.

We can rewrite, simplify, and partially solve the equations (20).

Theorem B. In the situation of Theorem $A$, let $w_{1}, \ldots, w_{n}, \theta$ satisfy (20). Write $w_{j} \equiv r_{j} e^{i \phi_{j}}$ and $\phi=\sum_{j=1}^{n} \phi_{j}$, for functions $r_{j}: I \rightarrow(0, \infty)$ 
and $\phi_{1}, \ldots, \phi_{n}, \phi: I \rightarrow \mathbb{R}$ or $\mathbb{R} / 2 \pi \mathbb{Z}$. Fix $s_{0} \in I$. Define $u: I \rightarrow \mathbb{R}$ by

$$
u(s)=2 \int_{s_{0}}^{s} r_{1}(t) \cdots r_{n}(t) \cos (\phi(t)-\theta(t)) \mathrm{d} t .
$$

Then $r_{j}^{2}(s) \equiv \alpha_{j}+\lambda_{j} u(s)$ for $j=1, \ldots, n$ and $s \in I$, where $\alpha_{j}=r_{j}^{2}\left(s_{0}\right)$. Define a degree $n$ polynomial $Q(u)$ by $Q(u)=\prod_{j=1}^{n}\left(\alpha_{j}+\lambda_{j} u\right)$. Then the system of equations (20) can be rewritten as

$$
\left\{\begin{aligned}
\frac{\mathrm{d} u}{\mathrm{~d} s} & =2 Q(u)^{1 / 2} \cos (\phi-\theta), \\
\frac{\mathrm{d} \phi_{j}}{\mathrm{~d} s} & =-\frac{\lambda_{j} Q(u)^{1 / 2} \sin (\phi-\theta)}{\alpha_{j}+\lambda_{j} u}, \quad j=1, \ldots, n, \\
\frac{\mathrm{d} \phi}{\mathrm{d} s} & =-Q(u)^{1 / 2}(\ln Q(u))^{\prime} \sin (\phi-\theta), \\
\frac{\mathrm{d} \theta}{\mathrm{d} s} & =\alpha Q(u)^{1 / 2} \sin (\phi-\theta) .
\end{aligned}\right.
$$

The Lagrangian self-similar solution $L$ in Theorem A may be rewritten

$$
\begin{array}{r}
L=\left\{\left(x_{1} \sqrt{\alpha_{1}+\lambda_{1} u(s)} e^{i \phi_{1}(s)}, \ldots, x_{n} \sqrt{\alpha_{n}+\lambda_{n} u(s)} e^{i \phi_{n}(s)}\right):\right. \\
\left.x_{1}, \ldots, x_{n} \in \mathbb{R}, s \in I, \sum_{j=1}^{n} \lambda_{j} x_{j}^{2}=C\right\} .
\end{array}
$$

Moreover, for some $A \in \mathbb{R}$ the equations (26) have the first integral

$$
Q(u)^{1 / 2} e^{\alpha u / 2} \sin (\phi-\theta) \equiv A .
$$

Proof. Using equations (20) and (26), for $j=1, \ldots, n$ we have

$$
\begin{aligned}
\frac{\mathrm{d}\left(r_{j}^{2}\right)}{\mathrm{d} s} & =\frac{\mathrm{d}\left(\left|w_{j}\right|^{2}\right)}{\mathrm{d} s}=w_{j} \frac{\mathrm{d} \bar{w}_{j}}{\mathrm{~d} s}+\bar{w}_{j} \frac{\mathrm{d} w_{j}}{\mathrm{~d} s}=\lambda_{j} e^{-i \theta} w_{1} \cdots w_{n}+\lambda_{j} e^{i \theta} \frac{\overline{w_{1}} \cdots w_{n}}{} \\
& =2 \lambda_{j} \operatorname{Re}\left(e^{i(\phi-\theta)} r_{1} \cdots r_{n}\right)=2 \lambda_{j} \cos (\phi-\theta) r_{1} \cdots r_{n}=\lambda_{j} \frac{\mathrm{d} u}{\mathrm{~d} s} .
\end{aligned}
$$

Thus $r_{j}^{2}-\lambda_{j} u$ is constant in $I$, and at $s=s_{0}$ we have $r_{j}^{2}\left(s_{0}\right)=\alpha_{j}$ and $u\left(s_{0}\right)=0$, so $r_{j}^{2}(s) \equiv \alpha_{j}+\lambda_{j} u(s)$ for $j=1, \ldots, n$ and $s \in I$, as we have to prove.

Differentiating (25) gives $\frac{\mathrm{d} u}{\mathrm{~d} s}=2 r_{1} \cdots r_{n} \cos (\phi-\theta)$. But $r_{j}^{2}=\alpha_{j}+$ $\lambda_{j} u$ and the definition of $Q$ imply that $Q(u)=\prod_{j=1}^{n} r_{j}^{2}$, so $\frac{\mathrm{d} u}{\mathrm{~d} s}=$ $2 Q(u)^{1 / 2} \cos (\phi-\theta)$, the first equation of (26). As $w_{j}=r_{j} e^{i \phi_{j}}$ we have

$$
\frac{\mathrm{d} w_{j}}{\mathrm{~d} s}=\frac{\mathrm{d} r_{j}}{\mathrm{~d} s} e^{i \phi_{j}}+i r_{j} e^{i \phi_{j}} \frac{\mathrm{d} \phi_{j}}{\mathrm{~d} s} .
$$

Thus $r_{j} \frac{\mathrm{d} \phi_{j}}{\mathrm{~d} s}=\operatorname{Im}\left(e^{-i \phi_{j}} \frac{\mathrm{d} w_{j}}{\mathrm{~d} s}\right)$, and the second equation of (26) follows from the first equation of (20), $w_{j}=r_{j} e^{i \phi_{j}}, \phi=\sum_{j=1}^{n} \phi_{j}$ and $Q(u)^{1 / 2}=$ $r_{1} \cdots r_{n}$. 
Summing the second equation of $(26)$ over $j=1, \ldots, n$ gives

$$
\frac{\mathrm{d} \phi}{\mathrm{d} s}=-Q(u)^{1 / 2} \sum_{j=1}^{n} \frac{\lambda_{j} \sin (\phi-\theta)}{\alpha_{j}+\lambda_{j} u}=-Q(u)^{1 / 2}(\ln Q(u))^{\prime} \sin (\phi-\theta),
$$

the third equation of (26). The final equation of (26) follows from the second equation of $(21), w_{j}=r_{j} e^{i \phi_{j}}, \phi=\sum_{j=1}^{n} \phi_{j}$ and $Q(u)^{1 / 2}=$ $r_{1} \cdots r_{n}$. Equation (27) is immediate from (21) and $w_{j}=r_{j} e^{i \phi_{j}}$. Finally, using (26) we find that

$$
\begin{aligned}
& \frac{\mathrm{d}}{\mathrm{d} s}\left(Q(u)^{1 / 2} e^{\alpha u / 2} \sin (\phi-\theta)\right)=\frac{1}{2} Q(u)^{1 / 2} \ln (Q(u))^{\prime} \frac{\mathrm{d} u}{\mathrm{~d} s} e^{\alpha u / 2} \sin (\phi-\theta) \\
& +Q(u)^{1 / 2} \frac{\alpha}{2} e^{\alpha u / 2} \frac{\mathrm{d} u}{\mathrm{~d} s} \sin (\phi-\theta)+Q(u)^{1 / 2} e^{\alpha u / 2} \cos (\phi-\theta) \frac{\mathrm{d}(\phi-\theta)}{\mathrm{d} s} \\
& =Q(u)^{1 / 2} e^{\alpha u / 2}\left[\sin (\phi-\theta)\left(\frac{1}{2} \ln (Q(u))^{\prime}+\frac{\alpha}{2}\right) 2 Q(u)^{1 / 2} \cos (\phi-\theta)\right. \\
& \left.+\cos (\phi-\theta)\left(-Q(u)^{1 / 2}(\ln Q(u))^{\prime} \sin (\phi-\theta)-\alpha Q(u)^{1 / 2} \sin (\phi-\theta)\right)\right]=0 .
\end{aligned}
$$

So the left-hand side of (28) is a constant, say, $A$ in $\mathbb{R}$.

q.e.d.

\section{Self-expanders diffeomorphic to $\mathcal{S}^{n-1} \times \mathbb{R}$}

We now prove Theorems C and D of $\S 3.2$.

Theorem C. In Theorems $A$ and B, suppose that $\lambda_{1}=\cdots=\lambda_{n}=$ $C=1, \alpha \geqslant 0$ and $A<0$. Then any solution of (20), or equivalently of (26), on an interval $I$ in $\mathbb{R}$ can be extended to a unique largest open interval $I_{\max }$ in $\mathbb{R}$. Take $I=I_{\max }$. Then by changing variables from $s$ in $I_{\max }$ to $y=y(s)$ in $\mathbb{R}$, we may rewrite the Lagrangian self-expander $L$ of (21) and (27) explicitly as follows. Conversely, every $L$ of the following form comes from Theorems $A$ and $B$ with $\lambda_{1}=\cdots=\lambda_{n}=C=1$, $\alpha \geqslant 0$ and $A<0$.

For given constants $\alpha \geqslant 0, a_{1}, \ldots, a_{n}>0$ and $\psi_{1}, \ldots, \psi_{n} \in \mathbb{R}$, define $w_{j}(y)=e^{i \phi_{j}(y)} r_{j}(y)$ for $j=1, \ldots, n$ and $y \in \mathbb{R}$ by

$$
\begin{gathered}
r_{j}(y)=\sqrt{\frac{1}{a_{j}}+y^{2}} \text { and } \phi_{j}(y)=\psi_{j}+\int_{0}^{y} \frac{\mathrm{d} t}{\left(\frac{1}{a_{j}}+t^{2}\right) \sqrt{P(t)}}, \\
\text { where } P(t)=\frac{1}{t^{2}}\left(\prod_{k=1}^{n}\left(1+a_{k} t^{2}\right) e^{\alpha t^{2}}-1\right) . \quad \text { Then } \\
L=\left\{\left(x_{1} w_{1}(y), \ldots, x_{n} w_{n}(y)\right): x_{1}, \ldots, x_{n} \in \mathbb{R}, \sum_{j=1}^{n} x_{j}^{2}=1\right\}
\end{gathered}
$$

is a closed, embedded Lagrangian diffeomorphic to $\mathcal{S}^{n-1} \times \mathbb{R}$ and satisfying $\alpha F^{\perp}=H$. If $\alpha>0$ it is a self-expander, and if $\alpha=0$ it is one of Lawlor's examples of special Lagrangian submanifolds [15]. It has Lagrangian angle

$$
\theta(y)=\sum_{j=1}^{n} \phi_{j}(y)+\arg \left(y+i P(y)^{-1 / 2}\right) .
$$


Proof. Suppose we are in the situation of Theorems A and B, with $\lambda_{1}=\cdots=\lambda_{n}=C=1, \alpha \geqslant 0, A<0$ and $I=I_{\max }$. Define $G(u)=$ $Q(u) e^{\alpha u}$ and $\beta=-\min _{j=1, \ldots, n} \frac{\alpha_{j}}{\lambda_{j}}<0$. Then $G(\beta)=0$, and

$$
\frac{\mathrm{d}}{\mathrm{d} u} \ln (G(u))=\sum_{j=1}^{n} \frac{\lambda_{j}}{\alpha_{j}+\lambda_{j} u}+\alpha,
$$

which is positive for $u>\beta$. Therefore $G$ is an increasing function on $[\beta, \infty)$ with $G(\beta)=0$ and $\lim _{u \rightarrow \infty} G(u)=\infty$. Note that $G(0)=$ $\prod_{j=1}^{n} \alpha_{j} \geqslant A^{2}$, since $(28)$ at $s=s_{0}$ gives $\left(\prod_{j=1}^{n} \alpha_{j}\right)^{1 / 2} \sin (\phi-\theta)=A$ and $|\sin (\phi-\theta)| \leqslant 1$. Hence there exists $u_{*} \in(\beta, 0]$ with $G\left(u_{*}\right)=A^{2}$ (see Figure 1).

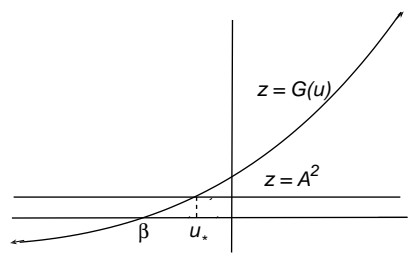

Figure 1. the case that $\lambda_{1}=\cdots=\lambda_{n}=C=1, \alpha \geqslant 0$, and $A<0$.

Since $|\sin (\phi-\theta)| \leqslant 1$, equation (28) implies that $G(u(s)) \geqslant A^{2}$ for all $s \in I$, and so $u(s) \geqslant u_{*}$ for all $s \in I$. Also, combining (26) and (28), we have $\frac{\mathrm{d} u}{\mathrm{~d} s}=0 \Leftrightarrow \cos (\phi-\theta)=0 \Leftrightarrow|\sin (\phi-\theta)|=1 \Leftrightarrow G(u(s))=A^{2}$ $\Leftrightarrow u(s)=u_{*}$. If $u$ is a constant function, then $u(s) \equiv 0$ as we have $u\left(s_{0}\right)=0$, and also $\phi-\theta \equiv-\frac{\pi}{2}$ as $A<0$. From (26) and (28), it follows that $\phi_{j}=\psi_{j}-\frac{A s}{\alpha_{j}}$ and $\theta=\theta_{0}+\alpha A s$ for some constants $\psi_{j}$ and $\theta_{0}$. As $\alpha_{j}>0$ and $\alpha \geqslant 0$, it contradicts to the fact that $\phi-\theta \equiv-\frac{\pi}{2}$. Now suppose $s_{1}, s_{2}$ are distinct, adjacent zeroes of $\frac{\mathrm{d} u}{\mathrm{~d} s}$ in $I$. Then $\frac{\mathrm{d} u}{\mathrm{~d} s}$ has constant sign in $\left(s_{1}, s_{2}\right)$, but $u\left(s_{1}\right)=u\left(s_{2}\right)=u_{*}$, giving a contradiction by the Mean Value Theorem. Hence $\frac{\mathrm{d} u}{\mathrm{~d} s}$ has at most one zero in $I$.

Write $I=(a, b)$ for $a, b \in \mathbb{R} \cup\{ \pm \infty\}$. We claim that $u(s) \rightarrow \infty$ as $s \rightarrow a_{+}$or $s \rightarrow b_{-}$. When $a$ or $b$ are finite this follows from $I=I_{\max }$, since the only way the solution could not extend over $a$ or $b$ is if $u \rightarrow \infty$. When $a$ or $b$ are infinite, $u$ is monotone near infinity as $\frac{\mathrm{d} u}{\mathrm{~d} s}$ has at most one zero, so $u(s)$ must approach infinity or some finite limiting value $u^{\prime}$ as $s \rightarrow \pm \infty$. If $u(s) \rightarrow u^{\prime}$ as $s \rightarrow \pm \infty$ then $\frac{\mathrm{d} u}{\mathrm{~d} s} \rightarrow 0$ as $s \rightarrow \infty$, forcing $u^{\prime}=u_{*}$ from above. We can exclude this possibility by showing that $\frac{\mathrm{d}^{2} u}{\mathrm{~d} s^{2}} \not \rightarrow 0$ as $s \rightarrow \infty$. Thus $u(s) \rightarrow \infty$ as $s \rightarrow a_{+}$or $s \rightarrow b_{-}$, and $u$ has at least one minimum at $s_{*}$ in $I$. Then $\frac{\mathrm{d} u}{\mathrm{~d} s}\left(s_{*}\right)=0$, so $s_{*}$ is unique from above, and $u\left(s_{*}\right)=u_{*}$. 
Combining the first equation of (26), (28), and the fact that $u$ is decreasing in $\left(a, s_{*}\right)$ and increasing in $\left(s_{*}, b\right)$, we have

$$
\frac{\mathrm{d} u}{\mathrm{~d} s}=\left\{\begin{aligned}
-2 \sqrt{Q(u)-A^{2} e^{-\alpha u}}, & a<s \leqslant s_{*}, \\
2 \sqrt{Q(u)-A^{2} e^{-\alpha u}}, & s_{*} \leqslant s<b .
\end{aligned}\right.
$$

This gives

$$
\int_{u_{*}}^{u(s)} \frac{\mathrm{d} v}{2 \sqrt{Q(v)-A^{2} e^{-\alpha v}}}=\left|s-s_{*}\right| .
$$

Thus $a, b$ are finite when $n>2$ and $a=-\infty, b=+\infty$ when $n \leqslant 2$.

Equation (35) defines $s$ explicitly as a function of $u$. Inverting this gives $u$ as a function of $s$. Then from (28) we obtain $\sin (\phi-\theta)$, and hence $\cos (\phi-\theta)$, as functions of $s$. Thus we have the right-hand side of each equation in (26) as functions of $s$, and integrating (26) gives $\phi_{j}, \phi$ and $\theta$ as functions of $s$.

To make this more explicit, not depending on inverting the integral function (35), we shall change from $s$ to a new variable $y$ defined by

$$
y(s)=\left\{\begin{aligned}
-\sqrt{u-u\left(s_{*}\right)}, & a<s \leqslant s_{*}, \\
\sqrt{u-u\left(s_{*}\right)}, & s_{*} \leqslant s<b .
\end{aligned}\right.
$$

Then $y:(a, b) \rightarrow \mathbb{R}$ is a smooth diffeomorphism, and $u=u\left(s_{*}\right)+y^{2}$, so that $r_{j}^{2}=\alpha_{j}+u=\alpha_{j}+u\left(s_{*}\right)+y^{2}=a_{j}^{-1}+y^{2}$, where $a_{j}=\left(\alpha_{j}+u\left(s_{*}\right)\right)^{-1}$. This gives $r_{j}(y)=\sqrt{\frac{1}{a_{j}}+y^{2}}$, as in (29). Computing $\frac{\mathrm{d} \phi_{j}}{\mathrm{~d} y}$ from $\frac{\mathrm{d} \phi_{j}}{\mathrm{~d} s}$ and $\frac{\mathrm{d} y}{\mathrm{~d} s}$ yields $\frac{\mathrm{d} \phi_{j}}{\mathrm{~d} y}=\left(\left(\frac{1}{a_{j}}+y^{2}\right) \sqrt{P(y)}\right)^{-1}$, for $P(y)$ as in (30). This implies the second equation of (29), with $\psi_{j}=\left.\phi_{j}\right|_{y=0}=\left.\phi_{j}\right|_{s=s_{*}}$.

Theorems A and B now imply that $L$ is Lagrangian with $\alpha F^{\perp}=H$, with Lagrangian angle (32). Equation (31) implies that $L$ is diffeomorphic to $\mathcal{S}^{n-1} \times \mathbb{R}$, and closedness of $L$ follows from $r_{j}(y) \rightarrow \pm \infty$ as $y \rightarrow \pm \infty$. That $L$ is embedded follows from the fact that each $\phi_{j}(y)$ is strictly increasing, and has image an interval of size at most $\pi$, as we will show in the proof of Theorem $\mathrm{D}$. When $\alpha=0$ our formulae reduce to those of Harvey's treatment [8, p. 139-143] of Lawlor's examples [15]. This completes the proof.

q.e.d.

Theorem D. In the situation of Theorem $C$, there exist $\bar{\phi}_{1}, \ldots, \bar{\phi}_{n} \in$ $\left(0, \frac{\pi}{2}\right]$ with $\bar{\phi}_{j}=\int_{0}^{\infty} \frac{\mathrm{d} t}{\left(\frac{1}{a_{j}}+t^{2}\right) \sqrt{P(t)}}$ for $j=1, \ldots, n$, such that the Lagrangian $L$ is asymptotic at infinity to the union of Lagrangian planes $L_{1} \cup L_{2}$, where

$$
\begin{aligned}
& L_{1}=\left\{\left(e^{i\left(\psi_{1}+\bar{\phi}_{1}\right)} t_{1}, \ldots, e^{i\left(\psi_{n}+\bar{\phi}_{n}\right)} t_{n}\right): t_{1}, \ldots, t_{n} \in \mathbb{R}\right\}, \\
& L_{2}=\left\{\left(e^{i\left(\psi_{1}-\bar{\phi}_{1}\right)} t_{1}, \ldots, e^{i\left(\psi_{n}-\bar{\phi}_{n}\right)} t_{n}\right): t_{1}, \ldots, t_{n} \in \mathbb{R}\right\} .
\end{aligned}
$$

We have $0<\bar{\phi}_{1}+\cdots+\bar{\phi}_{n}<\frac{\pi}{2}$ if $\alpha>0$, and $\bar{\phi}_{1}+\cdots+\bar{\phi}_{n}=\frac{\pi}{2}$ if $\alpha=0$. 
Fix $\alpha>0$. Then $\Phi^{n}:\left(a_{1}, \ldots, a_{n}\right) \mapsto\left(\bar{\phi}_{1}, \ldots, \bar{\phi}_{n}\right)$ gives a diffeomorphism

$$
\Phi^{n}:(0, \infty)^{n} \longrightarrow\left\{\left(\bar{\phi}_{1}, \ldots, \bar{\phi}_{n}\right) \in\left(0, \frac{\pi}{2}\right]^{n}: 0<\bar{\phi}_{1}+\cdots+\bar{\phi}_{n}<\frac{\pi}{2}\right\} .
$$

That is, for all $\alpha>0$ and $L_{1}, L_{2}$ satisfying $0<\bar{\phi}_{1}+\cdots+\bar{\phi}_{n}<\frac{\pi}{2}$ as above, Theorem $C$ gives a unique Lagrangian expander $L$ asymptotic to $L_{1} \cup L_{2}$.

When $\alpha=0$, it is studied by Lawlor in $[\mathbf{1 5}]$. The map $\Phi^{n}:\left(a_{1}, \ldots, a_{n}\right)$ $\mapsto\left(\bar{\phi}_{1}, \ldots, \bar{\phi}_{n}\right)$ gives a surjection

$$
\Phi^{n}:(0, \infty)^{n} \longrightarrow\left\{\left(\bar{\phi}_{1}, \ldots, \bar{\phi}_{n}\right) \in\left(0, \frac{\pi}{2}\right)^{n}: \bar{\phi}_{1}+\cdots+\bar{\phi}_{n}=\frac{\pi}{2}\right\},
$$

such that $\left(a_{1}, \ldots, a_{n}\right)$ and $\left(a_{1}^{\prime}, \ldots, a_{n}^{\prime}\right)$ have the same image $\left(\bar{\phi}_{1}, \ldots, \bar{\phi}_{n}\right)$ if and only if $a_{j}^{\prime}=t a_{j}$ for some $t>0$ and all $j=1, \ldots, n$, and the corresponding special Lagrangians $L, L^{\prime}$ satisfy $L^{\prime}=t^{-1 / 2} L$.

Proof. From the definition of $\phi_{j}(y)$ in (29), it is clear that the integral converges as $y \rightarrow \infty$ which is denoted by $\bar{\phi}_{j}>0$. Here $\bar{\phi}_{j}$ depends on $a_{1}, \ldots, a_{n}>0$ and $\alpha \geqslant 0$. The limit of the integral as $y \rightarrow-\infty$ is then $-\bar{\phi}_{j}$. This shows that $L$ is asymptotic to $L_{1} \cup L_{2}$. It is also easy to see that when $\alpha>0$, the convergence of $L$ to $L_{1} \cup L_{2}$ at infinity is exponential.

Since $P(y)^{-1 / 2}>0$ and $|y| \gg P(y)^{-1 / 2}$ for large $y$ by (30), we see that $\lim _{y \rightarrow-\infty} \arg \left(y+i P(y)^{-1 / 2}\right)=\pi$ and $\lim _{y \rightarrow \infty} \arg \left(y+i P(y)^{-1 / 2}\right)=0$. Thus (32) implies that

$$
\lim _{y \rightarrow-\infty} \theta(y)=\sum_{j=1}^{n} \psi_{j}-\sum_{j=1}^{n} \bar{\phi}_{j}+\pi, \quad \lim _{y \rightarrow \infty} \theta(y)=\sum_{j=1}^{n} \psi_{j}+\sum_{j=1}^{n} \bar{\phi}_{j} .
$$

But the last equation of (26), (28), and $A<0$ imply that $\theta$ is strictly decreasing when $\alpha>0$ and constant when $\alpha=0$. Hence $\lim _{y \rightarrow-\infty} \theta(y)>$ $\lim _{y \rightarrow \infty} \theta(y)$ when $\alpha>0$ and $\lim _{y \rightarrow-\infty} \theta(y)=\lim _{y \rightarrow \infty} \theta(y)$ when $\alpha=0$. By (39), this gives $\sum_{j=1}^{n} \bar{\phi}_{j}<\frac{\pi}{2}$ when $\alpha>0$, and $\sum_{j=1}^{n} \bar{\phi}_{j}=\frac{\pi}{2}$ when $\alpha=0$. As each $\bar{\phi}_{k}>0$, this implies that $\bar{\phi}_{j} \leqslant \frac{\pi}{2}$, and completes the first part.

Write the map $\Phi^{n}$ in the theorem as $\Phi^{n}=\left(\Phi_{1}^{n}, \ldots, \Phi_{n}^{n}\right)$. Then (29)(30) and the definition of $\Phi^{n}$ imply that

$$
\Phi_{j}^{n}\left(a_{1}, \ldots, a_{n}\right)=\int_{0}^{\infty} \frac{a_{j} \mathrm{~d} y}{\left(1+a_{j} y^{2}\right) \sqrt{\frac{1}{y^{2}}\left(\prod_{l=1}^{n}\left(1+a_{l} y^{2}\right) e^{\alpha y^{2}}-1\right)}}
$$

Computation shows that for $y \in(0, \infty)$ we have

$$
\frac{\partial}{\partial a_{k}}\left[\frac{a_{j}}{\left(1+a_{j} y^{2}\right) \sqrt{\frac{1}{y^{2}}\left(\prod_{l=1}^{n}\left(1+a_{l} y^{2}\right) e^{\alpha y^{2}}-1\right)}}\right]<0 \quad \text { for } k \neq j .
$$


Integrating this over $(0, \infty)$ and using (40) thus gives

$$
\frac{\partial}{\partial a_{k}}\left(\Phi_{j}^{n}\left(a_{1}, \ldots, a_{n}\right)\right)<0 \text { for } k \neq j .
$$

Let $t>0$. Replacing $a_{j}$ by $t a_{j}$ for $j=1, \ldots, m$ in (40) and changing variables from $y$ to $t^{-1 / 2} y$ shows that

$$
\Phi_{j}^{n}\left(t a_{1}, \ldots, t a_{n}\right)=\int_{0}^{\infty} \frac{a_{j} \mathrm{~d} y}{\left(1+a_{j} y^{2}\right) \sqrt{\frac{1}{y^{2}}\left(\prod_{l=1}^{n}\left(1+a_{l} y^{2}\right) e^{\left.t^{-1} \alpha y^{2}-1\right)}\right.}} .
$$

The integrand here is a strictly increasing function of $t$ for $\alpha>0$, and constant for $\alpha=0$. Thus, $\frac{\mathrm{d}}{\mathrm{d} t} \Phi_{j}^{n}\left(t a_{1}, \ldots, t a_{n}\right)$ is positive for $\alpha>0$ and zero for $\alpha=0$. Setting $t=1$ yields

$$
\sum_{k=1}^{n} a_{k} \frac{\partial}{\partial a_{k}}\left(\Phi_{j}^{n}\left(a_{1}, \ldots, a_{n}\right)\right) \begin{cases}>0, & \alpha>0, \\ =0, & \alpha=0 .\end{cases}
$$

Combining (41) and (43) implies that

$$
\frac{\partial}{\partial a_{j}}\left(\Phi_{j}^{n}\left(a_{1}, \ldots, a_{n}\right)\right)>0 .
$$

Also, taking the limit $t \rightarrow \infty$ in (42) we see that $\lim _{t \rightarrow \infty} \Phi_{j}^{n}\left(t a_{1}, \ldots, t a_{n}\right)$ exists, and equals $\bar{\phi}_{j}$ with the same $a_{1}, \ldots, a_{n}$ but with $\alpha=0$. But we have already shown that $\bar{\phi}_{1}+\cdots+\bar{\phi}_{n}=\frac{\pi}{2}$ when $\alpha=0$. Therefore

$$
\sum_{j=1}^{n} \lim _{t \rightarrow \infty} \Phi_{j}^{n}\left(t a_{1}, \ldots, t a_{n}\right)=\frac{\pi}{2} .
$$

Fixing $\alpha>0$, we first show that the differential of $\Phi^{n}$ is nonsingular. Suppose there exist $\lambda_{1}, \ldots, \lambda_{n} \in \mathbb{R}$ not all zero such that for $j=1, \ldots, n$ we have $\sum_{k=1}^{n} \lambda_{k} \frac{\partial}{\partial a_{k}}\left(\Phi_{j}^{n}\left(a_{1}, \ldots, a_{n}\right)\right)=0$. Pick $j$ such that $\left|\lambda_{j}\right| / a_{j}$ is greatest. Then (41) and (43) imply that

$$
\begin{aligned}
a_{j} \frac{\partial}{\partial a_{j}}\left(\Phi_{j}^{n}\left(a_{1}, \ldots, a_{n}\right)\right) & >-\sum_{k \neq j} a_{k} \frac{\partial}{\partial a_{k}}\left(\Phi_{j}^{n}\left(a_{1}, \ldots, a_{n}\right)\right) \text { and } \\
\frac{\left|\lambda_{j}\right|}{a_{j}} a_{j} \frac{\partial}{\partial a_{j}}\left(\Phi_{j}^{n}\left(a_{1}, \ldots, a_{n}\right)\right) & >-\sum_{k \neq j} \frac{\left|\lambda_{k}\right|}{a_{k}} a_{k} \frac{\partial}{\partial a_{k}}\left(\Phi_{j}^{n}\left(a_{1}, \ldots, a_{n}\right)\right) .
\end{aligned}
$$

It follows that

$$
\left|\lambda_{j} \frac{\partial}{\partial a_{j}}\left(\Phi_{j}^{n}\left(a_{1}, \ldots, a_{n}\right)\right)\right|>\left|\sum_{k \neq j} \lambda_{k} \frac{\partial}{\partial a_{k}}\left(\Phi_{j}^{n}\left(a_{1}, \ldots, a_{n}\right)\right)\right|,
$$

contradicting $\sum_{k=1}^{n} \lambda_{k} \frac{\partial}{\partial a_{k}}\left(\Phi_{j}^{n}\left(a_{1}, \ldots, a_{n}\right)\right)=0$. Thus no such $\lambda_{1}, \ldots, \lambda_{n}$ exist, and $\left.\mathrm{d} \Phi^{n}\right|_{\left(a_{1}, \ldots, a_{n}\right)}: \mathbb{R}^{n} \rightarrow \mathbb{R}^{n}$ is invertible. So $\Phi^{n}$ in (37) is a local diffeomorphism. The same argument when $\alpha=0$ shows that the only possible $\left(\lambda_{1}, \ldots, \lambda_{n}\right)$ are multiples of $\left(a_{1}, \ldots, a_{n}\right)$. So in (38), $\left.\mathrm{d} \Phi^{n}\right|_{\left(a_{1}, \ldots, a_{n}\right)}: \mathbb{R}^{n} \rightarrow \mathbb{R}^{n-1}$ has kernel $\left\langle\left(a_{1}, \ldots, a_{n}\right)\right\rangle$, and is surjective.

We will now show that when $\alpha>0$, the map $\Phi^{n}$ of (37) is surjective. Embed the domain $(0, \infty)^{n}$ of $\Phi^{n}$ in $\mathbb{R} \mathbb{P}^{n}$ by $\left(a_{1}, \ldots, a_{n}\right) \mapsto$ $\left[1, a_{1}, \ldots, a_{n}\right]$. The closure of $(0, \infty)^{n}$ in $\mathbb{R P}^{n}$ is an $n$-simplex $\Delta^{n}$. It has faces $\Delta_{j}^{n-1}$ for $j=0, \ldots, n$ on which $x_{j}=0$ in homogeneous coordinates $\left[x_{0}, \ldots, x_{n}\right]$. The closure in $\mathbb{R}^{n}$ of the range of $\Phi^{n}$ in (37) is also an 
$n$-simplex $\tilde{\Delta}^{n}$ with faces $\tilde{\Delta}_{j}^{n-1}$ for $j=0, \ldots, n$, where $\bar{\phi}_{1}+\cdots+\bar{\phi}_{n}=\frac{\pi}{2}$ on $\tilde{\Delta}_{0}^{n-1}$ and $\bar{\phi}_{j}=0$ on $\tilde{\Delta}_{j}^{n-1}$ for $j=1, \ldots, n$. Note that $\partial \Delta^{n}=$ $\cup_{j=0}^{n} \Delta_{j}^{n-1}$ and $\partial \tilde{\Delta}^{n}=\cup_{j=0}^{n} \tilde{\Delta}_{j}^{n-1}$.

We claim that $\Phi^{n}$ extends to a continuous map $\bar{\Phi}^{n}: \Delta^{n} \rightarrow \tilde{\Delta}^{n}$, which maps $\Delta_{j}^{n-1} \rightarrow \tilde{\Delta}_{j}^{n-1}$ for $j=0, \ldots, n$. To see this, note that $\lim _{a_{j} \rightarrow 0} \Phi_{k}^{n}\left(a_{1}, \ldots, a_{n}\right)$ exists for all $k$ by $(40)$, and $\lim _{a_{j} \rightarrow 0} \Phi_{j}^{n}\left(a_{1}, \ldots, a_{n}\right)=0$.

Thus $\Phi^{n}$ extends continuously to $\Delta_{j}^{n-1}$ for $j=1, \ldots, n$, and maps $\Delta_{j}^{n-1} \rightarrow \tilde{\Delta}_{j}^{n-1}$. Also, the fact above that $\lim _{t \rightarrow \infty} \Phi_{j}^{n}\left(t a_{1}, \ldots, t a_{n}\right)$ exists shows that $\Phi^{n}$ extends to $\Delta_{0}^{n-1}$, and (45) implies that this extension maps $\Delta_{0}^{n-1} \rightarrow \tilde{\Delta}_{0}^{n-1}$.

We will prove surjectivity of (37) and its extension $\bar{\Phi}^{n}$ by induction on $n$. The map is clearly onto when $n=1$ since it is continuous and $\bar{\Phi}^{1}([1,0])=0, \bar{\Phi}^{1}([0,1])=\frac{\pi}{2}$ by $(45)$. Suppose $\bar{\Phi}^{n-1}$ is surjective. Since $\bar{\Phi}^{n}$ reduces to $\bar{\Phi}^{n-1}$ when $a_{k}=0$, this implies that $\left.\bar{\Phi}^{n}\right|_{\Delta_{k}^{n-1}}$ : $\Delta_{k}^{n-1} \rightarrow \tilde{\Delta}_{k}^{n-1}$ is surjective for $k=1, \ldots, n$. Now consider $\left.\bar{\Phi}^{n}\right|_{\Delta_{0}^{n-1}}$ : $\Delta_{0}^{n-1} \rightarrow \tilde{\Delta}_{0}^{n-1}$. Since $\left.\bar{\Phi}^{n}\right|_{\Delta_{k}^{n-1}}$ is surjective for $k=1, \ldots, n$, we see that $\left.\bar{\Phi}^{n}\right|_{\Delta_{0}^{n-1} \cap \Delta_{k}^{n-1}}: \Delta_{0}^{n-1} \cap \Delta_{k}^{n-1} \rightarrow \tilde{\Delta}_{0}^{n-1} \cap \tilde{\Delta}_{k}^{n-1}$ is surjective for $k=1, \ldots, n$. So $\bar{\Phi}^{n}$ takes $\partial \Delta_{0}^{n-1}$ surjectively to $\partial \tilde{\Delta}_{0}^{n-1}$, and is of degree one. Using algebraic topology, it follows that $\left.\bar{\Phi}^{n}\right|_{\Delta_{0}^{n-1}}: \Delta_{0}^{n-1} \rightarrow \tilde{\Delta}_{0}^{n-1}$ is surjective. Hence $\bar{\Phi}^{n}$ takes $\partial \Delta^{n}$ surjectively to $\partial \Delta^{n}$, and is of degree one, so again, $\Phi^{n}$ is surjective.

Therefore by induction, $\Phi^{n}$ in (37) is surjective for all $n$. But $\Phi^{n}$ is a local diffeomorphism, and extends to a map $\Delta^{n} \rightarrow \tilde{\Delta}^{n}$ taking $\partial \Delta^{n} \rightarrow$ $\partial \tilde{\Delta}^{n}$, so $\Phi^{n}$ is proper, and thus $\Phi^{n}$ is a covering map. As the domain of $\Phi^{n}$ is connected and the range simply-connected, it follows that $\Phi^{n}$ in (37) is a diffeomorphism, as we have to prove. The final part for (38) follows by a similar argument; one way to do it is to show that the restriction of $\Phi^{n}$ in $(38)$ to $\left\{\left(a_{1}, \ldots, a_{n}\right) \in(0, \infty)^{n}: a_{1}+\cdots+a_{n}=1\right\}$ is a diffeomorphism.

q.e.d.

In the last two parts of Theorem $\mathrm{D}$, the proof that $\Phi^{n}$ is surjective is based on Lawlor [15, Lemma 10].

\section{Other self-similar solutions}

Finally we prove Theorems E and F of $\S 3.3$.

Theorem E. In Theorems $A$ and B, suppose that either:

(a) $\lambda_{1}=\cdots=\lambda_{n}=C=1, \alpha<0$ and $A>0$; or

(b) $\lambda_{1}=\cdots=\lambda_{m}=1$ and $\lambda_{m+1}=\cdots=\lambda_{n}=-1$ for some $1 \leqslant m<$ $n, C=1, A>0$, and $\alpha \in \mathbb{R}$. 
Then solutions exist for all $s \in \mathbb{R}$, and we take $I=\mathbb{R}$. In each of cases (a), (b) we divide into two subcases:

(i) $\sum_{j=1}^{n} \frac{\lambda_{j}}{\alpha_{j}}+\alpha=0$ and $\alpha_{1} \cdots \alpha_{n}=A^{2}$; or

(ii) otherwise.

In case (i), we have explicit solutions to (26) and obtain

$$
\begin{gathered}
L=\left\{\left(x_{1} \sqrt{\alpha_{1}} e^{i\left(\psi_{1}-\lambda_{1} A s / \alpha_{1}\right)}, \ldots, x_{n} \sqrt{\alpha_{n}} e^{i\left(\psi_{n}-\lambda_{n} A s / \alpha_{n}\right)}\right):\right. \\
\left.x_{1}, \ldots, x_{n} \in \mathbb{R}, s \in \mathbb{R}, \sum_{j=1}^{n} \lambda_{j} x_{j}^{2}=1\right\},
\end{gathered}
$$

which is Hamiltonian stationary in addition to being self-similar, and invariant under a subgroup $\mathbb{R}$ or $\mathrm{U}(1)$ of diagonal matrices $\left\{\operatorname{diag}\left(e^{i \lambda_{1} t / \alpha_{1}}\right.\right.$, $\left.\left.\ldots, e^{i \lambda_{n} t / \alpha_{n}}\right): t \in \mathbb{R}\right\}$ in $\mathrm{U}(n)$.

In case (ii), $u$ and $\phi-\theta$ are periodic in $s$ with period $S>0$, and

$$
\begin{aligned}
u(s+S) & =u(s), & \phi_{j}(s+S) & =\phi_{j}(s)+\gamma_{j}, \\
\phi(s+S) & =\phi(s)+\sum_{j=1}^{n} \gamma_{j}, & \theta(s+S) & =\theta(s)+\sum_{j=1}^{n} \gamma_{j},
\end{aligned}
$$

for some $\gamma_{1}, \ldots, \gamma_{n} \in \mathbb{R}$ and all $s \in \mathbb{R}$. In case (b) with $\alpha=0$ we have $\theta(s) \equiv \theta(0)$ and $\sum_{j=1}^{n} \gamma_{j}=0$.

Before the proof of Theorem E, we first derive the following lemma and proposition:

Lemma 6.1. In the situation of Theorem $E, G(u)=Q(u) e^{\alpha u}$ has a unique critical point $u^{*}$ on the interval $\left(\beta_{1}, \beta_{2}\right)$, where $\beta_{1}<0<\beta_{2}$ are defined by

$$
\beta_{1}=\left\{\begin{array}{ll}
-\min _{1 \leq j \leq n} \alpha_{j}, & \text { in (a), } \\
-\min _{1 \leq j \leq m} \alpha_{j}, & \text { in (b), }
\end{array} \quad \beta_{2}= \begin{cases}\infty, & \text { in (a), } \\
\min _{m+1 \leq j \leq n} \alpha_{j}, & \text { in (b). }\end{cases}\right.
$$

Also $\lim _{u \rightarrow \beta_{1}} G(u)=\lim _{u \rightarrow \beta_{2}} G(u)=0, G(u)>0$ on $\left(\beta_{1}, \beta_{2}\right), G^{\prime}(u)>$ 0 on $\left(\beta_{1}, u^{*}\right), G^{\prime}(u)<0$ on $\left(u^{*}, \beta_{2}\right)$, and $u(s) \in\left(\beta_{1}, \beta_{2}\right)$ for all $s \in I$.

Proof. The first derivative of $\ln G$ is given in (33). Differentiating yields

$$
\frac{\mathrm{d}^{2}}{\mathrm{~d} u^{2}} \ln (G(u))=-\sum_{j=1}^{n} \frac{\lambda_{j}^{2}}{\left(\alpha_{j}+\lambda_{j} u\right)^{2}}<0 .
$$

Hence $\frac{\mathrm{d}}{\mathrm{d} u} \ln (G(u))$ is strictly decreasing, and $\frac{\mathrm{d}}{\mathrm{d} u} \ln (G(u))$ can have at most one zero in any interval on which $G(u)>0$ so $\ln (G(u))$ is defined. By definition of $G$ we see that $\lim _{u \rightarrow \beta_{1}} G(u)=\lim _{u \rightarrow \beta_{2}} G(u)=0$ and $G(u)>0$ on $\left(\beta_{1}, \beta_{2}\right)$. Thus $G$ must have a global maximum $u^{*}$ in $\left(\beta_{1}, \beta_{2}\right)$. Then $u^{*}$ is a zero of $\frac{\mathrm{d}}{\mathrm{d} u} \ln (G(u))$ in $\left(\beta_{1}, \beta_{2}\right)$, so $u^{*}$ is unique, and $G^{\prime}(u)>0$ on $\left(\beta_{1}, u^{*}\right)$ and $G^{\prime}(u)<0$ on $\left(u^{*}, \beta_{2}\right)$ follow as $\frac{\mathrm{d}^{2}}{\mathrm{~d} u^{2}} \ln (G(u))<$ 0 . Finally, since $r_{j}^{2}(s)=\alpha_{j}+\lambda_{j} u(s)>0$ for all $s \in I$ and $j=1, \ldots, n$, we see that $u(s) \in\left(\beta_{1}, \beta_{2}\right)$ for all $s \in I$ from (48).

q.e.d. 
Proposition 6.2. In case (ii), there exist unique, finite $u_{1}, u_{2}$ with $\beta_{1}<u_{1}<u^{*}<u_{2}<\beta_{2}$ and $G\left(u_{1}\right)=G\left(u_{2}\right)=A^{2}$ and $G(u)>A^{2}$ on $\left(u_{1}, u_{2}\right)$. We have $u(s) \in\left[u_{1}, u_{2}\right]$ for all $s \in I$, and solutions exist for all $s \in \mathbb{R}$.

Proof. Since $\sin ^{2}(\phi-\theta) \leqslant 1$, equation (28) implies that $G(u(s)) \geqslant A^{2}$ for all $s \in I$. By Lemma 6.1, if $s \in I$ then $u(s) \in\left(\beta_{1}, \beta_{2}\right)$, so $G(u(s)) \leqslant$ $G\left(u^{*}\right)$. Thus $G\left(u^{*}\right) \geqslant A^{2}$. If $G\left(u^{*}\right)=A^{2}$ this forces $u(s)=u^{*}$ for all $s \in I$, so $u^{*}=u\left(s_{0}\right)=0$, giving $G\left(u^{*}\right)=\alpha_{1} \cdots \alpha_{n}=A^{2}$, and $\left.\frac{\mathrm{d}}{\mathrm{d} u} \ln (G(u))\right|_{u=0}=0$, giving $\sum_{j=1}^{n} \frac{\lambda_{j}}{\alpha_{j}}+\alpha=0$ by (33). Thus $G\left(u^{*}\right)=A^{2}$ implies we are in case (i).

Since we restricted to case (ii), we have $G\left(u^{*}\right)>A^{2}$. So Lemma 6.1 and the Intermediate Value Theorem imply that there exist unique $u_{1} \in\left(\beta_{1}, u^{*}\right)$ and $u_{2} \in\left(u^{*}, \beta_{2}\right)$ with $G\left(u_{1}\right)=G\left(u_{2}\right)=A^{2}$, and that if $u \in\left(\beta_{1}, \beta_{2}\right)$ then $G(u) \geqslant A^{2}$ if and only if $u \in\left[u_{1}, u_{2}\right]$, with $G(u)>A^{2}$ on $\left(u_{1}, u_{2}\right)$. Thus $G(u(s)) \in\left[u_{1}, u_{2}\right]$ for all $s \in I$, by Lemma 6.1. This is illustrated in Figure 2.

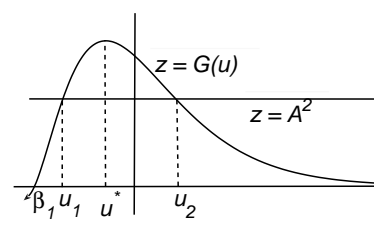

Figure 2. Case (a)

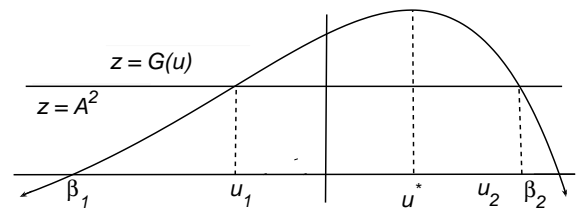

Case (b)

Suppose that in Theorem E we have solutions on some interval $I$ in $\mathbb{R}$. These must extend to some maximal open interval $I_{\max }=(a, b)$ in $\mathbb{R}$, for $a, b \in \mathbb{R} \cup\{ \pm \infty\}$. We could only have $a>-\infty$ if either $u(s) \rightarrow \infty$ as $s \rightarrow a_{+}$or $\alpha_{j}+\lambda_{j} u(s) \rightarrow 0$ as $s \rightarrow a_{+}$for some $j=1, \ldots, n$, so that the right-hand side of some equation in (26) becomes singular as $s \rightarrow a_{+}$, and the solutions do not extend past $a$. But this is impossible because it can only happen when $u(s)$ approaches $\beta_{1}$ or $\beta_{2}$, and $u(s)$ is confined to $\left[u_{1}, u_{2}\right]$, which lies strictly inside $\left(\beta_{1}, \beta_{2}\right)$ from the discussion above. Therefore $a=-\infty$, and similarly $b=\infty$, and solutions exist for all $s \in \mathbb{R}$.

q.e.d.

Now we are ready to prove Theorem E.

Proof of Theorem E. In case (i), $G(0)=G\left(u^{*}\right)=A^{2}$, equation (28) implies $\sin ^{2}(\phi-\theta) \equiv 1$. It is easy to verify that solutions to (26) are of the form

$$
\begin{gathered}
u(s)=0, \quad Q(u(s))=\alpha_{1} \cdots \alpha_{n}=A^{2}, \theta(s)=\sum_{j=1}^{n} \psi_{j}-\frac{\pi}{2}+\alpha A s, \\
\phi_{j}(s)=\psi_{j}-\frac{\lambda_{j} A s}{\alpha_{j}}, \phi(s)=\sum_{j=1}^{n} \psi_{j}-\sum_{j=1}^{n} \frac{\lambda_{j} A s}{\alpha_{j}}=\sum_{j=1}^{n} \psi_{j}+\alpha A s
\end{gathered}
$$


for some $\psi_{1}, \ldots, \psi_{n} \in \mathbb{R}$, which exist for all $s \in \mathbb{R}$. From (27) we obtain $L$ as described in (46) and have the induced metric on $L$ described in (22). Therefore, $\theta$ is harmonic and $L$ is Hamiltonian stationary.

In case (ii), we already proved that solutions exist for all $s \in \mathbb{R}$ in Proposition 6.2. It remains to show the solutions are periodic. The proof of (34) implies that

$$
\left(\frac{\mathrm{d} u}{\mathrm{~d} s}\right)^{2}=4\left(Q(u)-A^{2} e^{-\alpha u}\right)=4 e^{-\alpha u}\left(G(u)-A^{2}\right) .
$$

Thus $\frac{\mathrm{d} u}{\mathrm{~d} s}=0$ if and only if $G(u)=A^{2}$, that is, if and only if $u=u_{1}$ or $u=u_{2}$. So $\frac{\mathrm{d} u}{\mathrm{~d} s}$ cannot change sign except at $s$ with $u(s)=u_{1}$ or $u(s)=u_{2}$, and $\frac{\mathrm{d} u}{\mathrm{~d} s}$ is determined up to sign by $u(s)$. As for (35), the interval in $s$ taken for $u(s)$ to increase from $u_{1}$ to $u_{2}$, or to decrease from $u_{2}$ to $u_{1}$, is

$$
\frac{S}{2}=\int_{u_{1}}^{u_{2}} \frac{\mathrm{d} v}{2 \sqrt{Q(v)-A^{2} e^{-\alpha v}}},
$$

which is finite, since $G^{\prime}\left(u_{1}\right)>0$ and $G^{\prime}\left(u_{2}\right)<0$ by Lemma 6.1, so $Q(v)-A^{2} e^{-\alpha v}$ has only simple zeroes at $v=u_{1}$ and $v=u_{2}$.

Therefore $u$ is periodic with period $S>0$, as it must increase from $u=u_{1}$ to $u=u_{2}$ in an interval $S / 2$, then decrease back to $u=u_{1}$ in an interval $S / 2$, and repeat. Hence $\frac{\mathrm{d} u}{\mathrm{~d} s}$ has period $S$, so $\cos (\phi-\theta)$ is periodic with period $S$ by (26). Thus $\phi-\theta$ changes by an integral multiple of $2 \pi$ over each interval $S$. But (28) implies that $\sin (\phi-\theta)>0$, and $\phi-\theta$ is continuous, so this multiple of $2 \pi$ is zero, and $\phi-\theta$ is periodic with period $S$.

Equation (26) now implies that $\frac{\mathrm{d} \phi_{j}}{\mathrm{~d} s}$ is periodic with period $S$. Integrating gives $\phi_{j}(s+S)=\phi_{j}(s)+\gamma_{j}$ for all $j=1, \ldots, n$ and $s \in \mathbb{R}$, where $\gamma_{j}=\int_{0}^{S} \frac{\mathrm{d} \phi_{j}}{\mathrm{~d} s}(s) \mathrm{d} s$. Summing over $j=1, \ldots, n$ gives $\phi(s+S)=$ $\phi(s)+\sum_{j=1}^{n} \gamma_{j}$ for all $s \in \mathbb{R}$. Since $\phi-\theta$ is periodic with period $S$ this implies that $\theta(s+S)=\theta(s)+\sum_{j=1}^{n} \gamma_{j}$, proving (47). In case (b) with $\alpha=0$ we have $\frac{\mathrm{d} \theta}{\mathrm{d} s} \equiv 0$ by $(26)$, so $\theta(s) \equiv \theta(0)$, and $\sum_{j=1}^{n} \gamma_{j}=0$. This completes the proof of Theorem E. q.e.d.

Theorem F. In Theorem E, we say that $\left(w_{1}, \ldots, w_{n}\right)$ is periodic if there exists $T>0$ with $w_{j}(s)=w_{j}(s+T)$ for all $s \in \mathbb{R}$ and $j=1, \ldots, n$.

If $\left(w_{1}, \ldots, w_{n}\right)$ is periodic then in case $(\mathrm{a}), L$ is a compact, immersed Lagrangian self-shrinker diffeomorphic to $\mathcal{S}^{1} \times \mathcal{S}^{n-1}$, and in case (b), $L$ is a closed, noncompact, immersed Lagrangian diffeomorphic to $\mathcal{S}^{1} \times$ $\mathcal{S}^{m-1} \times \mathbb{R}^{n-m}$, a self-expander if $\alpha>0$, a self-shrinker if $\alpha<0$, and special Lagrangian if $\alpha=0$.

In case $(\mathrm{i}),\left(w_{1}, \ldots, w_{n}\right)$ is periodic if and only if $\frac{\lambda_{j}}{\alpha_{j}}=\mu q_{j}$ with $\mu>0$ and $q_{j} \in \mathbb{Q}$ for $j=1, \ldots, n$. In case (ii), $\left(w_{1}, \ldots, w_{n}\right)$ is periodic if and only if $\gamma_{j} \in \pi \mathbb{Q}$ for $j=1, \ldots, n$. In both cases, for fixed $m, \alpha$, there is a dense subset of initial data for which $\left(w_{1}, \ldots, w_{n}\right)$ is periodic. 
Proof. The first parts are straightforward. If $\left(w_{1}, \ldots, w_{n}\right)$ is periodic with period $T$ then $L$ is the image of an immersion $Q \times \mathbb{R} / T \mathbb{Z} \rightarrow \mathbb{C}^{n}$, where $Q$ is the quadric $\left\{\left(x_{1}, \ldots, x_{n}\right) \in \mathbb{R}^{n}: x_{1}^{2}+\cdots+x_{n}^{2}=1\right\}$ in (a), which is diffeomorphic to $\mathcal{S}^{n-1}$, and the quadric $\left\{\left(x_{1}, \ldots, x_{n}\right) \in \mathbb{R}^{n}\right.$ : $\left.x_{1}^{2}+\cdots+x_{m}^{2}-x_{m+1}^{2}-\cdots-x_{n}^{2}=1\right\}$ in (b), which is diffeomorphic to $\mathcal{S}^{m-1} \times \mathbb{R}^{n-m}$. Since $\mathbb{R} / T \mathbb{Z}$ is diffeomorphic to $\mathcal{S}^{1}, L$ is diffeomorphic as an immersed submanifold to $\mathcal{S}^{1} \times \mathcal{S}^{n-1}$ in (a), which is compact, and to $\mathcal{S}^{1} \times \mathcal{S}^{m-1} \times \mathbb{R}^{n-m}$ in (b), which is noncompact. It is a self-expander if $\alpha>0$, a self-shrinker if $\alpha<0$, and special Lagrangian if $\alpha=0$. We can also easily verify that $L$ is closed when $\left(w_{1}, \ldots, w_{n}\right)$ is periodic.

The necessary and sufficient conditions for periodicity in the last part are also easy. In case (i), if $\left(w_{1}, \ldots, w_{n}\right)$ is periodic with period $T$ then $e^{i \phi_{j}(s+T)}=e^{i \phi_{j}(s)}$ for $j=1, \ldots, n$, so (49) gives $\frac{\lambda_{j} A T}{\alpha_{j}} \in 2 \pi \mathbb{Z}$ for $j=1, \ldots, n$, and the condition holds with $\mu=\frac{2 \pi}{A T}>0$ and $q_{j}=$ $\frac{\lambda_{j} A T}{2 \pi \alpha_{j}} \in \mathbb{Z} \subset \mathbb{Q}$. Conversely, if $\frac{\lambda_{j}}{\alpha_{j}}=\mu q_{j}$ for $\mu>0$ and $q_{j} \in \mathbb{Q}$ then we may write $q_{j}=p_{j} / r$ for $j=1, \ldots, n, p_{j} \in \mathbb{Z}$ and $r \in \mathbb{N}$ the lowest common denominator of $q_{1}, \ldots, q_{n}$. Then $\left(w_{1}, \ldots, w_{n}\right)$ is periodic with period $\frac{2 \pi r}{A \mu}$.

In case (ii), since $u$ is periodic with period $S$, if $\left(w_{1}, \ldots, w_{n}\right)$ is periodic with period $T$ then $T=r S$ for some $r \in \mathbb{N}$. But then $e^{i \phi_{j}(s+T)}=e^{i \phi_{j}(s)}$ for $j=1, \ldots, n$, so (47) gives $e^{i r \gamma_{j}}=1$, and $\gamma_{j} \in 2 \pi \mathbb{Z} / r \subset \pi \mathbb{Q}$ for $j=1, \ldots, n$, as we want. Conversely, if $\gamma_{j} \in \pi \mathbb{Q}$ for $j=1, \ldots, n$ then we may write $\gamma_{j}=2 \pi p_{j} / r$ for $j=1, \ldots, n, p_{j} \in \mathbb{Z}$ and $r \in \mathbb{N}$, and then $\left(w_{1}, \ldots, w_{n}\right)$ is periodic with period $T=r S$.

It remains to show that in both cases, for fixed $m, \alpha$, there is a dense subset of initial data with $\left(w_{1}, \ldots, w_{n}\right)$ periodic. In case (i) this is straightforward: by Theorem E, for fixed $\lambda_{1}, \ldots, \lambda_{n}$ and $\alpha$, solutions are in 1-1 correspondence with choices of $\alpha_{1}, \ldots, \alpha_{n}>0$ and $\psi_{1}, \ldots, \psi_{n} \in \mathbb{R}$ satisfying $\sum_{j=1}^{n} \frac{\lambda_{j}}{\alpha_{j}}+\alpha=0$, and by the previous part, the corresponding solution is periodic if and only if $\frac{\lambda_{j}}{\alpha_{j}}=\mu q_{j}$ for $\mu>0$ and $q_{j} \in \mathbb{Q}$ for $j=1, \ldots, n$. It is easy to see that the set of such $\alpha_{j}, \psi_{j}$ is dense in the set of all allowed $\alpha_{j}, \psi_{j}$.

So we restrict to case (ii). In the special Lagrangian case $\alpha=0$, the first author $[\mathbf{1 2}, \S 5.5]$ showed that periodic solutions are dense in all solutions, so we suppose $\alpha \neq 0$. Given some solution in Theorem E, Lemma 6.1 found a unique $u^{*} \in\left[u_{1}, u_{2}\right] \subset\left(\beta_{1}, \beta_{2}\right)$ where $G(u)$ is maximum in $\left(\beta_{1}, \beta_{2}\right)$, and Theorem $\mathrm{E}$ showed that $u: \mathbb{R} \rightarrow \mathbb{R}$ cycles between $u_{1}$ and $u_{2}$ and so realizes all values in $\left[u_{1}, u_{2}\right]$, including $u^{*}$. Thus, in Theorem B we can choose the base point $s_{0} \in I=\mathbb{R}$ so that $u\left(s_{0}\right)=u^{*}$; effectively, this changes $\alpha_{j} \mapsto \alpha_{j}+\lambda_{j} u^{*}, u \mapsto u-u^{*}$, and $u^{*} \mapsto 0$. 
We will work for the rest of the proof with this normalization, so that $u^{*}=0$. Then $G(u)$ has a maximum at $u=0$, so that (33) gives

$$
\sum_{j=1}^{n} \frac{\lambda_{j}}{\alpha_{j}}+\alpha=0
$$

The remaining variables are $\alpha_{1}, \ldots, \alpha_{n}>0$ which satisfy (50), $A$ which satisfies $0<A<\left(\alpha_{1} \cdots \alpha_{n}\right)^{1 / 2}$ by (28) and (ii), and $\psi_{1}, \ldots, \psi_{n} \in$ $\mathbb{R}$. Now $\gamma_{1}, \ldots, \gamma_{n}$ are independent of $\psi_{1}, \ldots, \psi_{n}$, so we can regard them as functions of $\alpha_{1}, \ldots, \alpha_{n}$ and $A$. Define

$$
\begin{aligned}
& \Psi^{m, n}:\left\{\left(\alpha_{1}, \ldots, \alpha_{n}, A\right) \in(0, \infty)^{n+1}:\right. \\
& \left.\sum_{j=1}^{n} \frac{\lambda_{j}}{\alpha_{j}}+\alpha=0, A<\left(\alpha_{1} \cdots \alpha_{n}\right)^{1 / 2}\right\} \longrightarrow \mathbb{R}^{n}, \quad \text { where } \\
& \Psi^{m, n}=\left(\Psi_{1}^{m, n}, \ldots, \Psi_{n}^{m, n}\right):\left(\alpha_{1}, \ldots, \alpha_{n}, A\right) \longmapsto\left(\gamma_{1}, \ldots, \gamma_{n}\right) .
\end{aligned}
$$

To compute $\Psi_{j}^{m, n}$ explicitly, note that in one period $S$ of $s, u$ goes from $u_{1}$ up to $u_{2}$ and back down again, and $\psi_{j}$ increases by $\frac{\gamma_{j}}{2}$ in each half-period. So changing variables from $s$ to $u$ in $\left[u_{1}, u_{2}\right]$ we see that $\gamma_{j}=2 \int_{u_{1}}^{u_{2}} \frac{\mathrm{d} \phi_{j}}{\mathrm{~d} u}(u) \mathrm{d} u$, taking the branch of $\frac{\mathrm{d} \phi_{j}}{\mathrm{~d} u}$ on $\left(u_{1}, u_{2}\right)$ for which $\frac{\mathrm{d} u}{\mathrm{~d} s}>0$. Computing $\frac{\mathrm{d} \phi_{j}}{\mathrm{~d} u}$ from (26) and using (28) to eliminate terms in $\sin (\phi-\theta), \cos (\phi-\theta)$ gives

$$
\Psi_{j}^{m, n}\left(\alpha_{1}, \ldots, \alpha_{n}, A\right)=-\int_{u_{1}}^{u_{2}} \frac{A \lambda_{j} \mathrm{~d} v}{\left(\alpha_{j}+\lambda_{j} v\right) \sqrt{Q(v) e^{\alpha v}-A^{2}}},
$$

where $u_{1}<u^{*}=0<u_{2}$ are the closest roots of $Q(v) e^{\alpha v}=A^{2}$ to zero.

We must prove that $\Psi^{m, n}\left(\alpha_{1}, \ldots, \alpha_{n}, A\right) \in(\pi \mathbb{Q})^{n}$ for a dense subset of $\left(\alpha_{1}, \ldots, \alpha_{n}, A\right)$ in the domain of $\Psi^{m, n}$. To do this we will use the method of Joyce $[\mathbf{1 2}, \S 5.5]$. We first compute various limits of $\Psi^{m, n}$.

Proposition 6.3. Regarding $\alpha_{1}, \ldots, \alpha_{n}>0$ satisfying (50) as fixed, for all $j$ we have

$$
\lim _{A \rightarrow\left(\alpha_{1} \cdots \alpha_{n}\right)_{-}^{1 / 2}} \Psi_{j}^{m, n}\left(\alpha_{1}, \ldots, \alpha_{n}, A\right)=-2 \pi \lambda_{j} \alpha_{j}^{-1}\left(2 \sum_{k=1}^{n} \lambda_{k}^{2} \alpha_{k}^{-2}\right)^{-1 / 2} .
$$

Proof. Recall that $\left(\alpha_{1} \cdots \alpha_{n}\right)^{1 / 2} \sin (\phi-\theta)=A$ at $u=0$ from (7). When $A$ is close to $\left(\alpha_{1} \cdots \alpha_{n}\right)^{1 / 2}, u$ is small and $\sin (\phi-\theta)$ is close to 1 , so $\phi-\theta$ remains close to $\pi / 2$. Write $\phi-\theta=\frac{\pi}{2}+\varphi$, for $\varphi$ small. Then, setting $Q(u) \approx \alpha_{1} \cdots \alpha_{n}$,

$$
\cos (\phi-\theta) \approx-\varphi, \sin (\phi-\theta) \approx 1, \text { and } \sum_{k=1}^{n} \frac{\lambda_{k}}{\alpha_{k}+\lambda_{k} u}+\alpha \approx-u \sum_{k=1}^{n} \lambda_{k}^{2} \alpha_{k}^{-2}
$$


via linear approximation, taking only the highest order terms, equation (26) implies that

$$
\frac{\mathrm{d} u}{\mathrm{~d} s} \approx-2\left(\alpha_{1} \cdots \alpha_{n}\right)^{1 / 2} \varphi, \quad \frac{\mathrm{d}(\phi-\theta)}{\mathrm{d} s}=\frac{\mathrm{d} \varphi}{\mathrm{d} s} \approx u\left(\alpha_{1} \cdots \alpha_{n}\right)^{1 / 2} \sum_{k=1}^{n} \lambda_{k}^{2} \alpha_{k}^{-2} .
$$

It follows that

$$
\begin{aligned}
& \frac{\mathrm{d}^{2} u}{\mathrm{~d} s^{2}}+\left(2 \alpha_{1} \cdots \alpha_{n} \sum_{k=1}^{n} \lambda_{k}^{2} \alpha_{k}^{-2}\right) u \approx 0 \quad \text { and } \\
& \frac{\mathrm{d}^{2}(\phi-\theta)}{\mathrm{d} s^{2}}+\left(2 \alpha_{1} \cdots \alpha_{n} \sum_{k=1}^{n} \lambda_{k}^{2} \alpha_{k}^{-2}\right)(\phi-\theta) \approx 0,
\end{aligned}
$$

so that $u$ and $\phi-\theta$ undergo approximately simple harmonic oscillations with period $S=2 \pi\left(2 \alpha_{1} \cdots \alpha_{n} \sum_{k=1}^{n} \lambda_{k}^{2} \alpha_{k}^{-2}\right)^{-1 / 2}$. Then (26) shows that

$$
\frac{\mathrm{d} \phi_{j}}{\mathrm{~d} s} \approx-\lambda_{j} \alpha_{j}^{-1}\left(\alpha_{1} \cdots \alpha_{n}\right)^{1 / 2}
$$

which is approximately constant. Hence

$$
\gamma_{j}=\int_{0}^{S} \frac{\mathrm{d} \phi_{j}}{\mathrm{~d} s} \mathrm{~d} s \approx \frac{\mathrm{d} \phi_{j}}{\mathrm{~d} s} S=-2 \pi \lambda_{j} \alpha_{j}^{-1}\left(2 \sum_{k=1}^{n} \lambda_{k}^{2} \alpha_{k}^{-2}\right)^{-1 / 2}
$$

This proves (53).

q.e.d.

For an inductive step needed later, we have to compute what happens when $\alpha_{n} \rightarrow \infty$ or $\alpha_{1} \rightarrow \infty$ and include the case $m=0$ when $\alpha>0$, which is also well-defined using (51) and (52). So we allow $1 \leqslant m \leqslant n$ when $\alpha \leq 0$, and $0 \leqslant m<n$ when $\alpha>0$.

Proposition 6.4. Suppose $\left(\alpha_{1}(t), \ldots, \alpha_{n}(t), A(t)\right), t \in(1, \infty)$, is a continuous path in the domain of $\Psi^{m, n}$ in (51), such that

$$
\begin{gathered}
\lim _{t \rightarrow \infty} \alpha_{j}(t)=\tilde{\alpha}_{j} \quad \text { for } j=1, \ldots, n-1, \quad \lim _{t \rightarrow \infty} \alpha_{n}(t)=\infty, \\
\quad \text { and } \lim _{t \rightarrow \infty} A(t) \alpha_{n}(t)^{-1 / 2}=\tilde{A} .
\end{gathered}
$$

Then $\left(\tilde{\alpha}_{1}, \ldots, \tilde{\alpha}_{n-1}, \tilde{A}\right)$ is in the domain of $\Psi^{\tilde{m}, n-1}$, with $\tilde{m}=\min (m$, $n-1)$, and

$$
\lim _{t \rightarrow \infty} \Psi_{j}^{m, n}\left(\alpha_{1}(t), \ldots, \alpha_{n}(t), A(t)\right)= \begin{cases}\Psi_{j}^{\tilde{m}, n-1}\left(\tilde{\alpha}_{1}, \ldots, \tilde{\alpha}_{n-1}, \tilde{A}\right), & j<n, \\ 0, & j=n .\end{cases}
$$

Similarly, suppose $\left(\alpha_{1}(t), \ldots, \alpha_{n}(t), A(t)\right), t \in(1, \infty)$, is a continuous path in the domain of $\Psi^{m, n}$ such that

$$
\begin{gathered}
\lim _{t \rightarrow \infty} \alpha_{j}(t)=\tilde{\alpha}_{j} \quad \text { for } j=2, \ldots, n, \quad \lim _{t \rightarrow \infty} \alpha_{1}(t)=\infty, \\
\text { and } \quad \lim _{t \rightarrow \infty} A(t) \alpha_{1}(t)^{-1 / 2}=\tilde{A} .
\end{gathered}
$$


Then $\left(\tilde{\alpha}_{2}, \ldots, \tilde{\alpha}_{n}, \tilde{A}\right)$ is in the domain of $\Psi^{\tilde{m}-1, n-1}$, with $\tilde{m}=\max (m, 1)$, and

$$
\lim _{t \rightarrow \infty} \Psi_{j}^{m, n}\left(\alpha_{1}(t), \ldots, \alpha_{n}(t), A(t)\right)= \begin{cases}\Psi_{j-1}^{\tilde{m}-1, n-1}\left(\tilde{\alpha}_{2}, \ldots, \tilde{\alpha}_{n}, \tilde{A}\right), & j>1 \\ 0, & j=1\end{cases}
$$

Proof. We have $Q(v)=\prod_{j=1}^{n}\left(\alpha_{j}(t)+\lambda_{j} v\right)$. Write $\tilde{Q}(v)=\prod_{j=1}^{n-1}\left(\tilde{\alpha}_{j}+\right.$ $\left.\lambda_{j} v\right)$. Then in the case of (54) we see that the integrand in (52) satisfies

$$
\begin{aligned}
& \lim _{t \rightarrow \infty} \frac{A \lambda_{j}}{\left(\alpha_{j}+\lambda_{j} v\right) \sqrt{Q(v) e^{\alpha v}-A^{2}}} \\
& =\lim _{t \rightarrow \infty} \frac{1}{\alpha_{j}(t)+\lambda_{j} v} \lim _{t \rightarrow \infty} \frac{A(t)\left(\alpha_{n}(t)+\lambda_{n} v\right)^{-1 / 2} \lambda_{j}}{\sqrt{\prod_{l=1}^{n-1}\left(\alpha_{l}(t)+\lambda_{l} v\right) e^{\alpha v}-A(t)^{2}\left(\alpha_{n}(t)+\lambda_{n} v\right)^{-1}}} \\
& = \begin{cases}\frac{\tilde{A} \lambda_{j}}{\sqrt{\prod_{l=1}^{n-1}\left(\tilde{\alpha}_{l}+\lambda_{l} v\right) e^{\alpha v}-\tilde{A}^{2}}} \cdot \frac{1}{\tilde{\alpha}_{j}+\lambda_{j} v}, & j=1, \ldots, n-1, \\
0, & j=n .\end{cases}
\end{aligned}
$$

Applying the Dominated Convergence Theorem to (52), and noting that $u_{1}(t) \rightarrow \tilde{u}_{1}, u_{2}(t) \rightarrow \tilde{u}_{2}$, gives

$$
\begin{aligned}
\lim _{t \rightarrow \infty} \Psi_{j}^{m, n}\left(\alpha_{1}(t), \ldots, \alpha_{n}(t), A(t)\right) & =-\int_{\tilde{u}_{1}}^{\tilde{u}_{2}} \frac{\tilde{A} \lambda_{j} \mathrm{~d} v}{\left(\tilde{\alpha}_{j}+\lambda_{j} v\right) \sqrt{\tilde{Q}(v) e^{\alpha v}-\tilde{A}^{2}}} \\
& =\Psi_{j}^{\tilde{m}, n-1}\left(\tilde{\alpha}_{1}, \ldots, \tilde{\alpha}_{n-1}, \tilde{A}\right)
\end{aligned}
$$

if $j=1, \ldots, n-1$, and shows that the limit is 0 if $j=n$. This proves the first part of the proposition, and the second part is similar. q.e.d.

Note that when $\alpha>0$ and $m=n-1$, from (50) we always have $\alpha_{n}$ bounded. Similarly, when $\alpha<0$ and $m=1$ we always have $\alpha_{1}$ bounded. Hence the first and second parts of Proposition 6.4, respectively, cannot apply. In the following, we only need the first part of Proposition 6.4 when $\alpha<0$, and the second part when $\alpha>0$.

Proposition 6.5. For $\Psi^{m, n}$ as in (51) and (52), where we allow $m=n$ only if $\alpha<0$ and $m=0$ only if $\alpha>0$, the image Image $\Psi^{m, n}$ is $n$-dimensional, and for a dense open subset of $\left(\alpha_{1}, \ldots, \alpha_{n}, A\right)$ in the domain of $\Psi^{m, n}$, the following derivative is an isomorphism,

$$
\left.\mathrm{d} \Psi^{m, n}\right|_{\left(\alpha_{1}, \ldots, \alpha_{n}, A\right)}:\left\{\left(x_{1}, \ldots, x_{n}, y\right) \in \mathbb{R}^{n+1}: \sum_{j=1}^{n} \frac{\lambda_{j} x_{j}}{\alpha_{j}^{2}}=0\right\} \rightarrow \mathbb{R}^{n},
$$

so that $\Psi^{m, n}$ is a local diffeomorphism near $\left(\alpha_{1}, \ldots, \alpha_{n}, A\right)$.

Proof. By Proposition 6.3 the closure $\overline{\operatorname{Image} \Psi^{m, n}}$ contains the set

$$
\left\{\left(\frac{-2 \pi \lambda_{1} \alpha_{1}^{-1}}{\left(2 \sum_{k=1}^{n} \lambda_{k}^{2} \alpha_{k}^{-2}\right)^{1 / 2}}, \cdots, \frac{-2 \pi \lambda_{n} \alpha_{n}^{-1}}{\left(2 \sum_{k=1}^{n} \lambda_{k}^{2} \alpha_{k}^{-2}\right)^{1 / 2}}\right): \alpha_{j}>0 \text { for all } j\right\} \text {, }
$$


which is a nonempty open subset of the $(n-1)$-dimensional real hypersurface

$$
H=\left\{\left(\gamma_{1}, \ldots, \gamma_{n}\right) \in \mathbb{R}^{n}: \sum_{j=1}^{n} \gamma_{j}^{2}=2 \pi^{2}\right\}
$$

in $\mathbb{R}^{n}$. This implies that Image $\Psi^{m, n}$ is at least $(n-1)$-dimensional. Since $\Psi^{m, n}$ is real analytic and its domain is nonsingular and connected, there are only two possibilities:

(A) Image $\Psi^{m, n}$ is $n$-dimensional, or

(B) Image $\Psi^{m, n}$ lies in the $(n-1)$-dimensional real hypersurface $H$ in $\mathbb{R}^{n}$.

We shall use Proposition 6.4 and induction on $n$ to eliminate possibility (B), so that (A) holds. The first step $n=1$ is studied by Abresch and Langer [2], and translated into our notation, [2, Th. A \& Prop. 3.2(v)] implies that when $\alpha<0$ and $m=n=1$, Image $\Psi^{1,1}=(-\sqrt{2} \pi,-\pi)$. Changing signs of $\lambda_{1}, \alpha$ we deduce that when $\alpha>0, m=0$, and $n=1$, Image $\Psi^{0,1}=(\pi, \sqrt{2} \pi)$. Thus in both cases Image $\Psi^{m, n}$ is $n$-dimensional when $n=1$.

Suppose by induction that $n \geqslant 2$, and that Image $\Psi^{k, l}$ is $l$-dimensional whenever $l<n$, allowing $k=l$ only if $\alpha<0$, and $k=0$ only if $\alpha>0$. First suppose $\alpha<0$, and let $0<m \leqslant n$. Set $\tilde{m}=\min (m, n-$ $1)$. Then all of the domain of $\Psi^{\tilde{m}, n-1}$ arises as limits of the domain of $\Psi^{m, n}$ as in (54), so the first part of Proposition 6.4 implies that Image $\Psi^{\tilde{m}, n-1} \times\{0\} \subset \overline{\text { Image } \Psi^{m, n}}$. But by induction Image $\Psi^{\tilde{m}, n-1}$ is $(n-1)$-dimensional, so Image $\Psi^{\tilde{m}, n-1} \times\{0\}$ is not contained in the hypersurface $H$ in $\mathbb{R}^{n}$ because (Image $\left.\Psi^{\tilde{m}, n-1} \times\{0\}\right) \cap H$ is at most $(n-2)$ dimensional. So (B) does not hold.

Similarly, if $\alpha>0$ then for $0 \leqslant m<n$ and $\tilde{m}=\max (m, 1)$ the second part of Proposition 6.4 implies that $\{0\} \times \operatorname{Image} \Psi^{\tilde{m}-1, n-1} \subset$ $\overline{\text { Image } \Psi^{m, n}}$, and $\{0\} \times$ Image $\Psi^{\tilde{m}-1, n-1}$ is $(n-1)$-dimensional and not contained in $H$, so (B) does not hold. Thus in both cases (A) holds, so Image $\Psi^{m, n}$ is $n$-dimensional, proving the inductive step. The final parts follow as $\Psi^{m, n}$ is real analytic and its domain is nonsingular and connected.

q.e.d.

We can now complete the proof of Theorem F. By Proposition 6.5, $\Psi^{m, n}$ is a local diffeomorphism near $\left(\alpha_{1}, \ldots, \alpha_{n}, A\right)$ for $\left(\alpha_{1}, \ldots, \alpha_{n}, A\right)$ in a dense open subset $U$ in the domain of $\Psi^{m, n}$. As $(\pi \mathbb{Q})^{n}$ is dense in the range $\mathbb{R}^{n}$ of $\Psi^{m, n}$, it follows that $\left(\Psi^{m, n}\right)^{-1}\left((\pi \mathbb{Q})^{n}\right)$ is dense in $U$, and hence in the domain of $\Psi^{m, n}$, since $U$ is dense. But a choice of initial data gives a periodic solution if and only if $\Psi^{m, n}\left(\alpha_{1}, \ldots, \alpha_{n}, A\right) \in(\pi \mathbb{Q})^{n}$, by a previous part of the theorem. Since this holds for a dense subset of allowed $\left(\alpha_{1}, \ldots, \alpha_{n}, A\right)$, a dense subset of choices of initial data yield periodic solutions. 


\section{References}

[1] H. Anciaux, Construction of Lagrangian self-similar solutions to the mean curvature flow in $\mathbb{C}^{n}$, Geom. Dedicata 120 (2006), 37-48, MR 2252892, Zbl 1098.35074.

[2] U. Abresch \& J. Langer, The normalized curve shortening flow and homothetic solutions, J. Diff. Geom. 23 (1986), 175-196, MR 0845704, Zbl 0592.53002.

[3] K.A. Brakke, The motion of a surface by its mean curvature, Mathematical Notes, Princeton University Press, 1978, MR 0485012, Zbl 0386.53047.

[4] T. Bridgeland, Stability conditions on triangulated categories, Ann. of Math. 166 (2007), 317-345, MR 2373143, Zbl 1137.18008, math.AG/0212237.

[5] A. Butscher, Regularizing a singular special Lagrangian variety, Comm. Anal. Geom. 12 (2004), 733-791, MR 2104075, Zbl 1070.53026, math.DG/0110053.

[6] K. Fukaya, Y.-G. Oh, H. Ohta \& K. Ono, Lagrangian intersection Floer theory: anomaly and obstruction, Part I, AMS/IP Studies in Advanced Mathematics, 46.1, A.M.S., Providence, RI, MR 2553465, Zbl pre05616008.

[7] K. Fukaya, Y.-G. Oh, H. Ohta \& K. Ono, Lagrangian intersection Floer theory: anomaly and obstruction, Part II, AMS/IP Studies in Advanced Mathematics, 46.2, A.M.S., Providence, RI, MR 2548482, Zbl pre05616044.

[8] F.R. Harvey, Spinors and calibrations, Perspectives in Math. 9, Academic Press, San Diego, 1990, MR 1045637, Zbl 0694.53002.

[9] R. Harvey \& H.B. Lawson, Calibrated geometries, Acta Math. 148 (1982), 48156, MR 0666108, Zbl 0584.53021.

[10] G. Huisken, Asymptotic behavior for singularities of the mean curvature flow, J. Diff. Geom. 31 (1990), 285-299, MR 1030675, Zbl 0694.53005.

[11] G. Huisken \& C. Sinestrari, Convexity estimates for mean curvature flow and singularities of mean convex surfaces, Acta Math. 183 (1999), 45-70, MR 1719551, Zbl 0992.53051.

[12] D.D. Joyce, Constructing special Lagrangian $m$-folds in $\mathbb{C}^{m}$ by evolving quadrics, Math. Ann. 320 (2001), 757-797, MR 1857138, Zbl 1085.53503, math.DG/0008155.

[13] D.D. Joyce, Special Lagrangian submanifolds with isolated conical singularities. V. Survey and applications, J. Diff. Geom. 63 (2003), 279-347, MR 1857138, Zbl 1085.53503, math.DG/0608399.

[14] M. Kontsevich, Homological algebra of Mirror Symmetry, pages 120-139 in Proce. Int. Cong. Mathematicians, Vol. 1, (Zürich, 1994), Birkhäuser, Basel, 1995, MR 1403918, Zbl 0846.53021. alg-geom/9411018.

[15] G. Lawlor, The angle criterion, Inventiones math. 95 (1989), 437-446, MR 0974911, Zbl 0662.49018.

[16] D. Lee, Connected sums of special Lagrangian submanifolds, Comm. Anal. Geom. 12 (2004), 553-579, MR 2128603, Zbl 1064.53054.

[17] Y.-I. Lee, Embedded special Lagrangian submanifolds in Calabi-Yau manifolds, Comm. Anal. Geom. 11 (2003), 391-423, MR 2015752, Zbl 1099.53053.

[18] Y.-I. Lee \& M.-T. Wang, Hamiltonian stationary shrinkers and expanders for Lagrangian mean curvature flows, J. Diff. Geom. 83 (2009), 27-42, MR 2545029, Zbl pre05632055. 
[19] Y.-I. Lee \& M.-T. Wang, Hamiltonian stationary cones and self-similar solutions in higher dimension, Trans. Amer. Math. Soc. 362 (2010), 1491-1503. arXiv:0802.0359.

[20] A. Neves, Singularities of Lagrangian mean curvature flow: zero-Maslov class case, Invent. math. 168 (2007), 449-484, MR 2299559, Zbl 1119.53052 math.DG/0608399.

[21] A. Neves \& G. Tian, Translating solutions to Lagrangian mean curvature flow, arXiv:0711.4341, 2007.

[22] G. Perelman, The entropy formula for the Ricci flow and its applications, math.DG/0211159, 2002.

[23] R. Schoen \& J.G. Wolfson, Minimizing area among Lagrangian surfaces: the mapping problem, J. Diff. Geom. 58 (2001), 1-86, MR 1895348, Zbl 1052.53056, math.DG/0008244.

[24] K. Smoczyk, A canonical way to deform a Lagrangian submanifold, dgga/9605005, 1996.

[25] A. Strominger, S.-T. Yau \& E. Zaslow, Mirror symmetry is T-duality, Nuclear Phys. B479 (1996), 243-259, MR 1429831, Zbl 0896.14024. hep-th/9606040.

[26] R.P. Thomas \& S.-T. Yau, Special Lagrangians, stable bundles and mean curvature flow, Comm. Anal. Geom. 10 (2002), 1075-1113, MR 1429831, Zbl 0998.81091, math.DG/0104197.

[27] M.-T. Wang, Mean curvature flow of surfaces in Einstein four-manifolds, J. Diff. Geom. 57 (2001), 301-388, MR 1879229, Zbl 1035.53094, math.DG/0110019.

[28] B. White, The size of the singular set in mean curvature flow of mean-convex sets, J. Amer. Math. Soc. 13 (2000), 665-695, MR 1758759, Zbl 0961.53039.

[29] B. White, The nature of singularities in mean curvature flow of mean-convex sets, J. Amer. Math. Soc. 16 (2003), 123-138, MR 1937202, Zbl 1027.53078.

The Mathematical Institute, 24-29 St. Giles,

OXFORD, OX1 3LB, UK

E-mail address: joyce@maths.ox.ac.uk

Department OF MATHEMATICS AND Taida Institute of Mathematical Sciences,

National TaiWan University, TAIPEI 10617, TAIWAN

E-mail address: yilee@math.ntu.edu.tw

Department of Mathematics, UNIVERSITY OF TOLEDO,

TOLEDO, OH 43606, USA, AND Taida Institute of Mathematical Sciences, TAIPEI, TAIWAN

E-mail address: email: mao-pei.tsui@utoledo.edu 